\title{
Epitaxial exchange-bias systems: from fundamentals to future spin-orbitronics
}

\author{
Wei Zhang \\ Materials Sciences Division, Argonne National Laboratory, Argonne IL 60439 \\ zwei@anl.gov \\ Kannan M. Krishnan \\ Department of Materials Sciences \& Engineering, University of Washington, Seattle WA 98195 \\ kannanmk@uw.edu
}

(Dated: September 3, 16)

\begin{abstract}
Exchange bias has been investigated for more than half a century and several insightful reviews, published around the year 2000, have already summarized many key experimental and theoretical aspects related to this phenomenon. Since then, due to developments in thin-film fabrication and sophisticated characterization methods, exchange bias continues to show substantial advances; in particular, recent studies on epitaxial systems, which is the focus of this review, allow many long-standing mysteries of exchange bias to be unambiguously resolved. The advantage of epitaxial samples lies in the well-defined interface structures, larger coherence lengths, and competing magnetic anisotropies, which are often negligible in polycrystalline samples. Beginning with a discussion of the microscopic spin properties at the ferromagnetic/antiferromagnetic interface, we correlate the details of spin lattices with phenomenological anisotropies, and finally connect the two by introducing realistic measurement approaches and models. We conclude by providing a brief perspective on the future of exchange bias and related studies in the context of the rapidly evolving interest in antiferromagnetic spintronics.
\end{abstract}




\section{INTRODUCTION}

Exchange bias (EB) manifests itself as a shift of the center of the magnetic hysteresis loop from $H$ $=0$ to $H_{\mathrm{eb}} \neq 0$. It arises from the interfacial coupling of ferromagnetic(F)-antiferromagnetic(AF) heterostructures, under field cooling (FC) the sample below their respective Curie, $T_{\mathrm{C}}$, and Néel, $T_{\mathrm{N}}$, temperatures. The EB effect, along with its many supplemental physical phenomena, has been studied for many decades since its discovery by Meiklejohn and Bean [1], and motivated in part also by its array of technological applications. Several well-written review papers, published about fifteen years ago, offered a comprehensive understanding of EB, from experimental [2, 3], theoretical $[4,5]$, and nanoscale [6] perspectives. Interested readers should refer to these review articles, along with the lists of publications cited therein, for a general understanding of exchange bias. One of the most relevant elements in understanding EB is the interface structure. Practically all current theories for exchange bias assume or postulate a specific interface magnetic structure/coupling based on single crystals, such as in-plane collinear, perpendicular, or out-ofplane. More details on the theoretical models can be found in Ref. [4]. In reality, however, the interface structure is not necessarily identical to the bulk magnetic ordering even for single-crystal EB systems. To complicate matters even further, relaxation and reconstruction of either crystallographic or magnetic origins can develop on either side, i.e. AF or F, of the interface. In addition, even ten years ago the exact atomic arrangement in the vicinity of the interface was poorly resolved due to the lack of suitable experimental methods to probe the interface with atomic resolution. Such uncertainty is further compounded by the complexity of magnetic anisotropies, with many equivalent easy axes directions that are often present in epitaxial samples. 
Exchange bias and its related effects were investigated using experimental techniques available fifteen years ago: the most commonly used techniques were magnetization curve measurements using magnetometers, such as vibrating sample magnetometer (VSM) or superconducting quantum interference device (SQUID), and magneto-optic Kerr effect (MOKE). Other techniques such as torque magnetometry, ferromagnetic resonance (FMR), anisotropic magnetoresistance (AMR), and Mossbauer effect were also used. Various imaging techniques, such as magnetic force microscopy (MFM), Lorentz microscopy in a TEM, and Kerr microscopy, also allowed the study of EB by monitoring the ferromagnetic reversal behaviors. In their review article, published in 1999 [2], Nogues and Schuller summarized the key results for EB before year 2000 and presented a list of unresolved issues, which included: (1) thickness dependence (of F and AF), (2) AF orientation (uncompensated/compensated interfaces, out-of-plane spins), (3) interface disorder (roughness, crystallinity, grain size, interfacial impurity), (4) competing anisotropies, (5) blocking temperature, (6) training effect, (7) coercivity, (8) cooling field, and (9) perpendicular coupling. Since its publication, these unsolved issues have motivated much research during the past fifteen years. For example, O'Grady et al [7] systematically studied sputtered polycrystalline EB films and presented a new paradigm for exchange bias, focusing on the critical role played by the AF grains. Such phenomenological models have also shed light on the understanding of interface disorder, blocking temperature, coercivity, and training effect by incorporating the AF grain size and distribution; however, it does not readily apply to core-shell particles and epitaxial single-crystal films. Further, past studies focused primarily on the EB phenomenon itself but largely ignored the possible influences of different materials issues. For example, the roles of the intrinsic crystallinity of materials (as well as the additional and relevant anisotropies) were ignored since they have been poorly revealed in polycrystalline samples. Only recently, the importance of material crystallinity to EB has been recognized [8]; and there has been a renaissance in the field of EB by revisiting and studying optimized crystal orientations in old but prototypical materials, such as $\mathrm{CoO}$ [9], and other new materials. In addition, a complete understanding of the intrinsic spin behavior, interface coupling mechanisms, and AF anisotropy all require a high-quality $\mathrm{F} / \mathrm{AF}$ interface, because the essential behavior of EB critically depends on the atomic-level chemical and magnetic structure. In practice, these requirements can be satisfied in studies of epitaxial single-crystal films where both the interface quality and crystalline coherence are optimized. In particular, the spin configuration at the interfaces can be tuned by choosing different crystallographic orientations in epitaxial growth, and the role of interface 
roughness can be understood by changing the growth parameters [10]. Recent advances in thinfilm growth technology, such as ion-beam sputtering (IBS) and molecular-beam epitaxy (MBE), where the deposition rate can be controlled at the sub-monolayer level, have greatly promoted the fabrication of high quality epitaxial EB samples. Such epitaxial growth methods have been developed for AF metal-oxides (e.g. $\mathrm{CoO}, \mathrm{NiO})$ as well as metallic alloys (e.g. FeMn, IrMn and PdMn). Notably, epitaxial growth of alloys is more difficult since their crystal structures often deviate more strongly from cubic symmetry compared to the oxides. However, there are also advantages for using metallic alloys: (1) their spin structures are generally more complex, often involving multi-spin sublattices and/or temperature-dependent spin phases, which can give rise to novel magnetic properties; (2) the Néel temperatures of the metallic alloys are usually higher than that for insulating oxides, thus the contribution from the intrinsic AF anisotropy to the EB (may be established during sample growth), in addition to the field-cooling effect, can be investigated $[3,4]$. From the characterization point of view, in the past fifteen years, accesses to advanced techniques using $x$-rays (synchrotron radiation) [11], neutrons (scattering and reflectivity [12]) and photons (Brillouin light scattering [13]) have greatly increased, resulting in more persuasive and conclusive experimental data by direct spin-dependent measurements at the interface. For example, element-specific $x$-ray magnetic dichroism allows both $\mathrm{F}$ and AF spin properties to be studied independently and unambiguously. These advanced characterization methods exhibit significant advantage especially when applied to epitaxial single-crystal films, where a strong correlation between the intrinsic spin properties and exchange bias exists.

In this review, we present a summary of recent results, mostly after year 2000, on epitaxial EB samples. We discuss at length the two unique features in epitaxial samples, i.e. interface structures and competing anisotropies; without such, a full understanding of EB cannot be established by only working on polycrystalline samples. For the interface section, we first show that a much clearer scenario on the spin behaviors and coupling mechanisms at the interface can be obtained by studying epitaxial samples, with direct evidence coming from advanced characterization methods. Second, we discuss the critical role played by the enhanced AF crystallinity in epitaxial samples, including the various induced anisotropies and the effects from the long-range spin ordering. We demonstrate that competing anisotropies, with different origins and symmetries, give rise to exotic magnetization reversal processes, including reversal asymmetry, stepped hysteresis loops, nonaveraging effect, and mixed coherent-incoherent reversal mechanisms. We also summarize relevant theoretical works for understanding many of the unique behaviors of epitaxial EB 
samples, and demonstrate how these theories and models establish the connection between a 'difficult-to-resolve' interface spin configurations and the 'readily-measurable' magnetization reversal via phenomenological discussions of competing anisotropies. We conclude with an outlook on how the present study of epitaxial EB systems could shed light on the future developments in antiferromagnetic spintronics from a materials science perspective, including most recent advances such as new magnetoresistance and spin-orbital effects from antiferromagnets.

\section{EPITAXIAL EXCHANGE-Bias SySTEMS}

Epitaxial systems are important due to the fact that many EB related phenomena can be best observed and understood unambiguously by well-defined interface and bulk magnetic structures. Even though more than 60 years have elapsed since the discovery of EB in $\mathrm{Co} / \mathrm{CoO}$ particles, metal-oxides such as $\mathrm{CoO}$ and $\mathrm{NiO}$ remain popular $\mathrm{AF}$ candidates due to various advantages including well-defined magnetic structures, low Néel temperatures and high AF anisotropies. For example, epitaxial growth of $\mathrm{CoO}(001) / \mathrm{Fe}(001)$ [14], $\mathrm{FeNi}(111) / \mathrm{CoO}(111)$ [15], $\mathrm{Fe}_{3} \mathrm{O}_{4} / \mathrm{CoO}(001)$ $[16,17]$, and $\mathrm{CoFe} / \mathrm{NiO}(111)[18]$, or $\mathrm{Co} / \mathrm{NiO}(001)$ and $\mathrm{Fe} / \mathrm{NiO}(001)[19,20]$ have been demonstrated in the past few years. Besides metal-oxides, a significant amount of work has also focused on metallic-salt AFs in the past decade. Two facts have made such AF-alloys attractive as well as challenging: (1) The bi-metallic nature of these compounds makes the spin structures generally more complex, with multiple spin-lattices and possibly, temperature dependent phase transitions; in addition, the epitaxial growth of metallic alloys is usually more difficult. (2) The Néel temperature, $T_{\mathrm{N}}$, is usually higher than metal-oxides, and therefore, they may be good for potential device applications $[3,4]$. However, this also means that it is not always possible to fieldcool them through $T_{\mathrm{N}}$ without risking irreversible structural changes including grain growth and inter-diffusion. FeMn is a metallic alloy that has been widely studied in its polycrystalline form before 2000. Jungblut et al studied the orientation effect, in which a strong relationship has been established between the crystal orientations and the EB; however, there was no indication whether the compensation of the interface plays a role [21]. Liu et al looked at the interface roughness on $\mathrm{EB}$ in NiFe/FeMn [22] bilayers. Recently, epitaxial FeMn has also attracted much attention due to its triple-Q structure, featuring the triply degenerate points, $Q_{1}=(2 \pi / \mathrm{a})(1,0,0), Q_{2}=(2 \pi / \mathrm{a})(0,1,0)$, $Q_{3}=(2 \pi / \mathrm{a})(0,0,1)$, which is also known as the 'all-in-all-out' spin texture [23]. NiMn was investigated because of its good chemical and thermal stabilities [24, 25], and IrMn is well known 
as a disordered AF [26]. In particular, the out-of-plane EB systems utilizing IrMn (111) normal are favored over other crystallographic orientations due to the more efficient pinning and larger bias field. For example, large exchange bias, either perpendicular or in-plane, were reported extensively for IrMn/Co-based systems [27-31]. Such large bias fields were believed to be strongly locked with the composition and microstructure of both the IrMn and the ferromagnetic layers. Epitaxial growth with the IrMn (002) normal was only reported recently in systems with cubic symmetry [32-35] because of a good lattice match with many common ferromagnetic materials such as Fe and NiFe. The reported bias fields for these samples were usually a magnitude smaller than their polycrystalline counterparts, due to both the unfavorable spin orientation at the (002) plane and the subsequently reduced interface pinning. The annealing effect, which has been widely used in polycrystalline samples for enhancing the biasing, is also less significant for epitaxial grown samples [35].
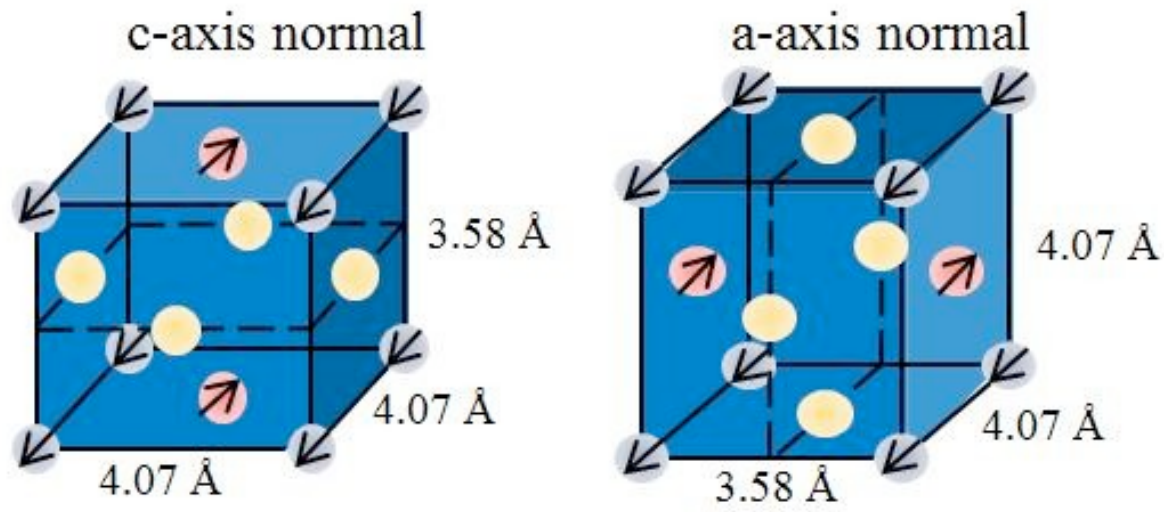

FIG. 1. (Color online) Schematic illustrating the epitaxial growth of MnPd unit cell along $c$-axis and $a$-axis normal directions; $c$-axis gives a compensated surface while $a$-axis gives an uncompensated surface. Blue and red spheres: Mn atoms with magnetic moment $\sim 4.4 \mu_{\mathrm{B}}$ arranged with opposite spin directions; yellow spheres: $\mathrm{Pd}$ atom with subtle (if not none) induced magnetic moments.

Investigation on the effects of AF crystallography on EB requires epitaxial growth of the AF films in their single crystal form, and preferably, with different crystallographic orientations along the film normal, while maintaining other parameters unmodified, such as the coherent crystallography of the $\mathrm{F}$ layer. Schuller and his colleagues have fabricated and studied epitaxial $\mathrm{Fe} / \mathrm{FeF}_{2}$ and $\mathrm{Fe} / \mathrm{MnF}_{2}$ bilayers with the $\mathrm{FeF}_{2}$ and $\mathrm{MnF}_{2}$ grown along different crystal orientations [36]. Another good material example is $\mathrm{MnPd}$, which has been successfully grown epitaxially on Fe with the MnPd along $c$ - or $a$-axis orientations $[10,37]$. MnPd is a chemically ordered L10 (CuAuI-type structure) antiferromagnetic alloy with $T_{\mathrm{N}}=540^{\circ} \mathrm{C}$ (bulk). In this structure, Mn has a magnetic moments of $4.4 \mu_{\mathrm{B}}$ (room temperature) with the easy axes along [100] and [ $\overline{100]}$ (or 
[010] and [0 $\overline{1} 0])$, while the moment is vanishingly small for Pd. The MnPd unit cell is facecentered-tetragonal $(f c t)$ with lattice parameters $a_{0}=b_{0}=4.07 \AA$ and $c_{0}=3.58 \AA$. By inspecting the spin structure of bulk MnPd, it is obvious that the (001) orientation gives a compensated surface, while (010) and (100) show an uncompensated surface (Fig. 1). Such differences in their surface compensation give rise to different $\mathrm{F} / \mathrm{AF}$ coupling and magnetic reversal properties once the $\mathrm{AF}$ is exchange-coupled to a $\mathrm{F}$ layer [38]. In addition, the lattice mismatch between $\mathrm{Fe}$ and $\mathrm{MnPd}$ is negligibly small $(\leq 1 \%)$ which makes it very suitable for investigating the bulk effect of EB.

\section{INTERFACE STRUCTURES}

The advantages of studying EB effects with epitaxial samples are the following: (1) Individual spin behavior and coupling mechanism can be revealed due to a high-quality interface (Fig. 2(b)); (2) AF crystallinity and interface AF domains play a role in the magnetization reversal due to the optimized structural coherence across the film (Fig. 2(c)). This effect is more significant when the scale of AF domains is comparable to, or even greater than, the F domains; (3) Bulk effect of the $\mathrm{AF}$ on the $\mathrm{EB}$ can be critical due to the long correlation length along the direction perpendicular to the interface (Fig. 2(d)). In epitaxial samples, the EB largely depends on the chemical and magnetic structures of the AF layer, which can also be strongly affected by defects, dislocations, and relaxation. All these factors, including AF spin behaviors, coupling mechanisms, interface compensation, AF crystallinity, surface terminating layer, and AF bulk effect are discussed in the following sections.

\subsection{Pinned and rotatable AF spins}

Practically all models of EB assume that the AF spins at the interface are pinned to establish the bias (loop shift), but experiments suggest that complete pinning of AF spins at the interface is neither true nor necessary for EB. For example, one important supplementary phenomenon of exchange bias is the enhanced loop coercivity [2]. Such enhancement has been attributed to a large part of the AF spins, buried under the interface, rotating together with F spins during the magnetization reversal. The relative quantities of pinned and rotatable spins are determined by intrinsic material properties such as the interfacial coupling strength and the AF anisotropy, as 

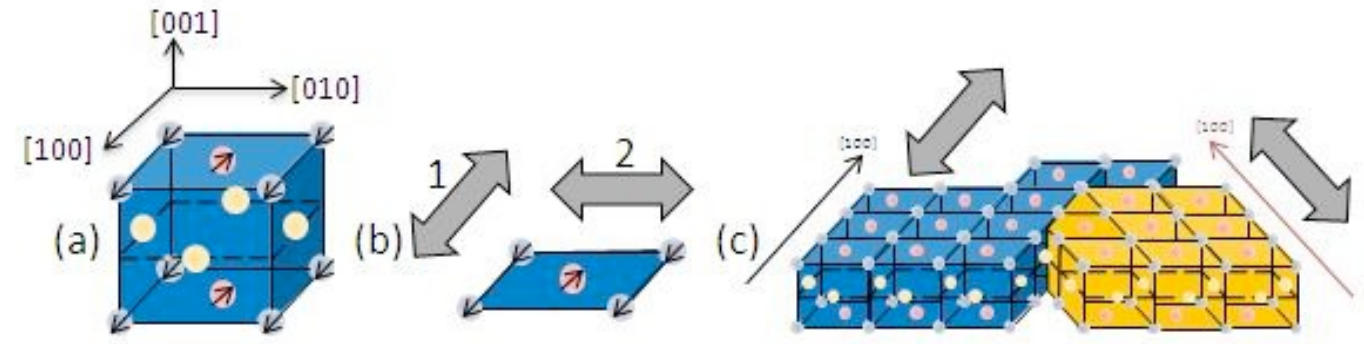

(d)

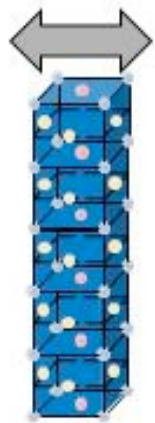

FIG. 2: (Color online) (a) High chemical ordering and well-defined interface structures in epitaxial samples offer unique factors influencing the EB effect but which are not effective in their polycrystalline counterparts. (b) Different orientations of the spin magnetic moment (1 and 2) with respect to the crystal lattice structure as well as their coupling mechanisms can be revealed. (c) Lateral arrangement of AF domains can play a role in EB. (d) Bulk effect along the direction perpendicular to the interface can be critical.

well as the extrinsic interface parameters, such as atomic steps [39] and roughness. Roy et al (2005) [40] performed polarized neutron and $x$-ray reflectivity on epitaxial $\mathrm{Co} / \mathrm{FeF}_{2} / \mathrm{MgF}_{2}(110)$

films and observed an uncompensated magnetization in the $\mathrm{FeF}_{2}$ layer that rotates in concert with the Co spins. Such rotatable magnetization in the AF was shown to be coupled antiparallel with Co spins and buried within $3.5 \mathrm{~nm}$ of the interface. Depth profile of the rotatable spins was also reported in epitaxial $\mathrm{CoO} / \mathrm{Fe} / \mathrm{Ag}(001)$ [41] using $x$-ray magnetic circular dichroism (XMCD), where a mixed layer of rotatable and pinned spins (2.2- $4.5 \mathrm{~nm}$ below the interface) was confirmed. Follow-up work showed that such pinned/rotatable behavior strongly depends on $\mathrm{CoO}$ thickness [42], where AF Co spins can be completely rotatable in ultra-thin $\mathrm{CoO}$ samples [43]. Similar experiments have been performed on other single crystal systems, e.g. $\mathrm{NiO} / \mathrm{Fe}_{3} \mathrm{O}_{4}$ [44]. In polycrystalline EB systems, the rotatable components are observed but they are less pronounced; e.g. in Permalloy/CoO [45], rotatable spins were detected but within a much thinner buried layer $(0.5 \mathrm{~nm})$ under the interface and in NiFe/IrMn [46], nearly no rotatable component was detected due to the chemical intermixing. In addition to synchrotron radiation, more accessible techniques also provide indirect evidence to the pinned and rotatable behavior, including tunneling magnetoresistance [47], MFM [48-50], and FMR [51-53].

Synchrotron radiation with element specificity is a powerful tool to directly reveal the AF spin behavior at the interface. For example, x-ray magnetic reflectometry (XRMR) characterization of $a$-axis $\mathrm{Fe} / \mathrm{MnPd}$ samples is shown in Fig. 3. The prerequisite for AF spins to rotate with $\mathrm{F}$ spins during $\mathrm{F}$ reversal is their strong exchange coupling at the interface. It was found that the hysteresis loops measured by element specific XRMR for Mn and Fe atoms are nearly 
overlapping, indicating a strong exchange coupling between the Fe and Mn moments during magnetic reversal. Further, quantitative analysis of the magnetic reflectivity spectra measured at the Fe $L$ and $\mathrm{Mn} L$ edges allows for a precise magnetic depth profiling of the interface region between the F and AF. It revealed rotatable, uncompensated Mn moments in a $4.1 \AA$ broad region at the $\mathrm{AF} / \mathrm{F}$ interface; further, the rotatable $\mathrm{Mn}$ and the ferromagnetic Fe couple antiparallel. In addition, a much broader $(\sim 13 \AA)$ region of pinned, uncompensated Mn moments, which couple parallel to the Fe moments during field cooling was found [54,55].

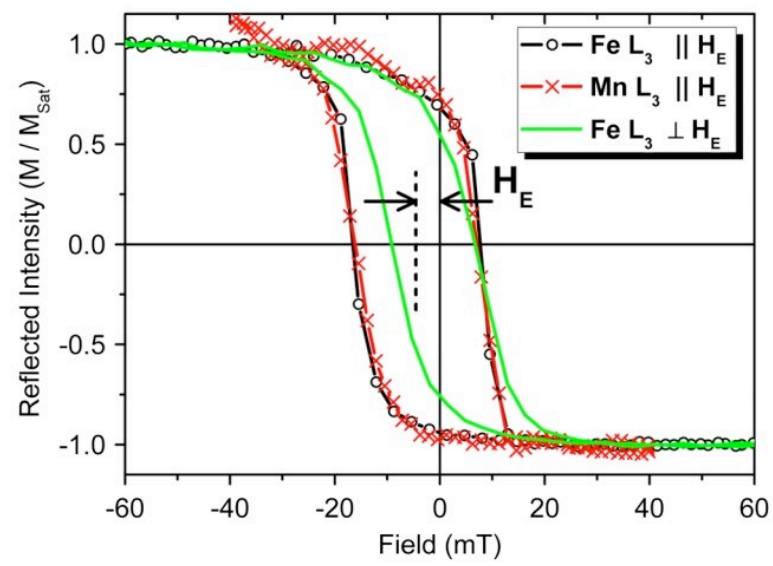

FIG. 3. (Color online) Hysteresis loops for Fe and Mn measured in an epitaxial Fe/MnPd bilayer by element specific XRMR [54]. The Mn atomic moment reproduces the same shape as that of Fe moment, when measured along the direction of the exchange bias, indicating a strong exchange coupling between the Fe and Mn moments during magnetic reversal. Reprinted from [54], copyright (2008), with permission from APS.

\subsection{Interface coupling mechanisms}

The nature of the coupling mechanism between interfacial $\mathrm{F}$ and AF spins determines the observed EB effect and related phenomena. Different coupling types have been theoretically postulated ten years ago and, to some extent, confirmed by preliminary magnetic reversal measurements. Theoretically, collinear couplings, either ferromagnetic (parallel) or antiferromagnetic (antiparallel), with respect to the F spin lattice, are proposed for uncompensated AF spins at the interface. On the other hand, perpendicular coupling is expected for a perfect compensated AF interface [56]. However, these different mechanisms can be complicated by possible interfacial spin-canting and roughness. Only in the past decade the true nature of the interfacial spin coupling has been directly revealed by advanced techniques. For example, the parallel F/AF coupling — one of the prevalent mechanisms - has been directly confirmed in $\mathrm{Co} / \mathrm{LaFeO}_{3} / \mathrm{SrTiO}_{3}(001)$ [57], $\mathrm{CoFe} / \mathrm{NiO}(111)$ [18], $\mathrm{Co} / \mathrm{NiO}(001)$ [58], $\mathrm{Co} / \mathrm{NiO}(100)$ [59], $\mathrm{NiO} / \mathrm{Fe}(001)$ [60], $\mathrm{MnPd}(001) / \mathrm{Fe} / \mathrm{MgO}(001)$ [61], $\mathrm{MnPd}(100) / \mathrm{Fe} / \mathrm{MgO}(001)$ [54, 55], 
$\operatorname{IrMn}(002) / \mathrm{Fe} / \mathrm{MgO}(001)$ [35], $\mathrm{NiO} / \mathrm{Fe}_{3} \mathrm{O}_{4}(001)$ [44], $\mathrm{FeMn} / \mathrm{Co}$ [62], etc. A more uncommon anti-parallel coupling was found in epitaxial $\mathrm{Co} / \mathrm{FeF}_{2} / \mathrm{MgF}_{2}(110)$ for both the pinned [63] and rotatable [40] AF spins, and also in perpendicular $\mathrm{CoO} / \mathrm{CoPt}$ multilayers [48]. Perpendicular coupling (spin-flop) has been found in epitaxial $\mathrm{CoO} / \mathrm{Fe} / \mathrm{Ag}(001)$ [41], $\mathrm{Co} / \mathrm{NiO}[19,64]$, $\mathrm{NiO} / \mathrm{Fe}(001)$ [20], $\mathrm{Fe} / \mathrm{NiO} / \mathrm{Ag}(001)$ [65], $\mathrm{Fe}_{3} \mathrm{O}_{4} / \mathrm{CoO}(001)$ [17]. Out-of-plane coupling has been found in epitaxial $\mathrm{FeMn} / \mathrm{Ni} / \mathrm{Cu}(001)$ [66]. It is worth noting that different coupling types can be observed in the same system by varying layer thicknesses and measurement temperatures. For example as shown in Fig. 4 , in the $a$-axis Fe/MnPd bilayers, the F/AF coupling is collinear for MnPd thickness $<30 \mathrm{~nm}$, but perpendicular for MnPd thickness $\geq 30 \mathrm{~nm}$, both measured at $20 \mathrm{~K}$ [67], well below the blocking temperature of MnPd. Such thickness-driven spin reorientation transition can be interpreted from the hysteresis loop measurements. The perpendicular coupling is characterized by a perpendicularly induced uniaxial anisotropy relative to the bias direction, which further results in a two-step reversal characteristic (Fig. 4(b)); in contrast to that the collinear uniaxial anisotropy and the one-step reversal can be seen in the parallel coupling case (Fig. 4(a)). Similar transitions can be also driven by temperature, i.e. even for the thicker sample $\left(t_{\mathrm{MnPd}} \geq 30 \mathrm{~nm}\right)$, the coupling is still collinear at $T>90 \mathrm{~K}\left(\sim T_{\mathrm{B}}\right.$ of $a$-axis Fe/MnPd), see Fig. 4(c). Such spin-reorientation-transition may be physically attributed to competing effects between the interface-induced-uniaxial anisotropy and intrinsic ferromagnetic anisotropy, especially if these two parameters were to have different thickness and/or temperature dependence, with one of the two overcoming the other when the system reaches certain critical values of thickness or temperature [68].
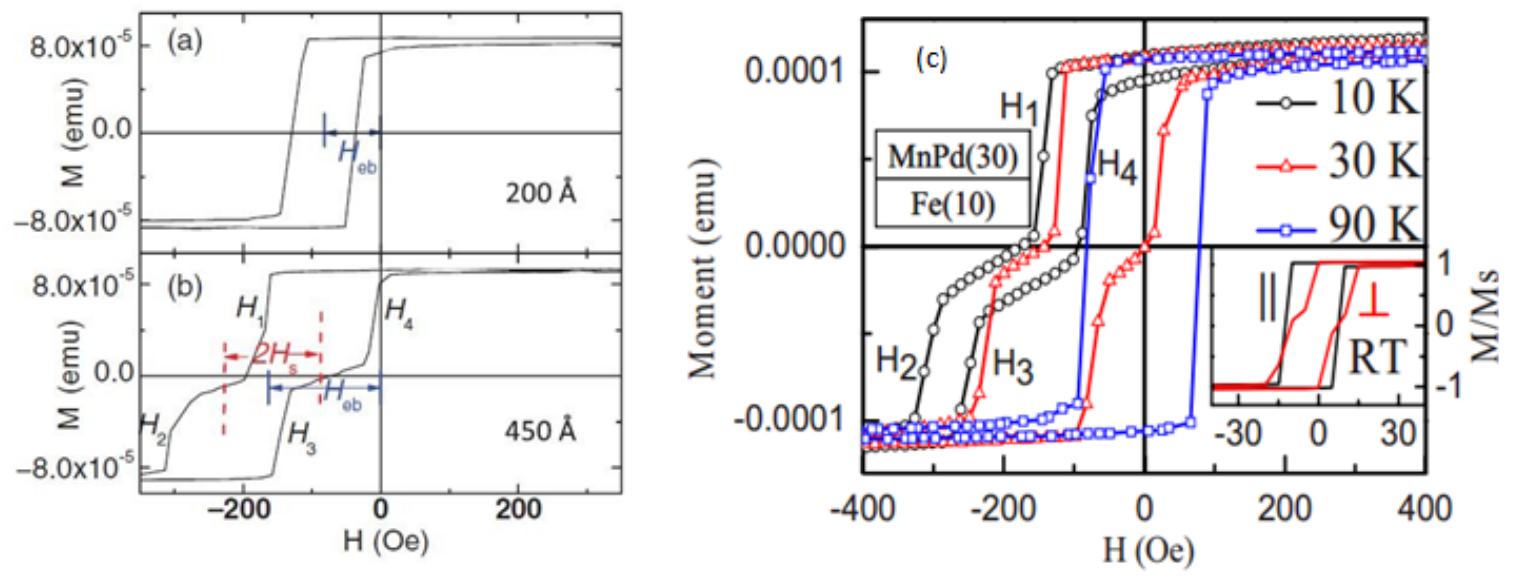

FIG. 4. (Color online) Hysteresis loop of spin-flop coupled Fe/MnPd epitaxial bilayer system for two MnPd thicknesses ( $t$ ) (a) $t=200 \AA$, and (b) $t=450 \AA$, at $20 \mathrm{~K}$. Reprinted from [67], copyright (2012), with permission from APS. (c) Temperature induced transition from collinear to spin-flop coupling for the same bilayer structure with $t=300 \AA$. Reprinted from [92], copyright (2010), with permission from AIP. 
These simple correlations, established between the type of coupling and nature of the AF spin compensation at the interface is also in agreement with the pioneering theoretical model by Koon [56], in which a perpendicular coupling is a natural consequence of a perfectly compensated AF interface due to the frustrated interaction between the spins on the F and the two AF sublattices. However, many experiments in the past a few years also showed sensitivity of the coupling to various practical film parameters that can overwhelm the intrinsic spin arrangements at the interface, and therefore, a case-by-case analysis is required. Parameters that can influence the nature of the coupling include, but are not limited to, film thickness [19, 42, 66, 69], atomic-steps and nature of the substrates [65, 70-73], interlayer spacer [74-77], terminating surface layers [78, 79], and temperature [67, 80]. Physically, it may involve either changing the AF anisotropy (film thickness and temperature), or modifications of the interfacial characteristics (atomic-steps, substrates, spacer, and layer termination). Last but not least, it has also been noted that in certain epitaxial bilayer systems, the uncompensated interfacial AF spins may also couple to the external field (higher energy barrier), which adds another competing interaction to their coupling to the $\mathrm{F}$ layer. As a result, extrinsic parameters such as field cooling may also play a role. Due to the competing coupling between AF-F and AF-external field, a positive (negative) EB can be established at lower (higher) cooling fields. Such an effect has been observed in the epitaxial $\mathrm{Fe} / \mathrm{FeF}_{2}$ and $\mathrm{Fe} / \mathrm{MnF}_{2}$ samples [81]. The uncompensated $\mathrm{AF}$ component, if large enough, can also result in a vertical shift of the hysteresis loop, with downward shifts indicating an antiferromagnetic coupling at the interface $[82,83]$.

\subsection{Compensated/uncompensated interface}

Another important parameter for EB is the AF interface spin arrangements (compensation). Earlier reviews [2-5] have already discussed the general picture of how the interface compensation would affect the EB-related properties. However, any effects related to the interface compensation are routinely averaged out for polycrystalline samples after removing the training effects. Practically, one of the advantages of epitaxial samples is the controllable, welldefined interfaces (compensated / uncompensated) by selective growth along different crystallographic orientations [84]. For example, Schuller et al have performed systematic studies on the $\mathrm{EB}$ of ferromagnets when coupled with single-crystal $\mathrm{FeF}_{2}$ or $\mathrm{MnF}_{2}$ antiferromagnets. Prototypical compensated [(110) and (101)] and uncompensated [(100) and (001)] interfaces were achieved [36]. Cheng et al., Gatel et al. and Krug et al. have also done systematic work in this regard for $\mathrm{Fe} / \mathrm{MnPd}[37]$ and $\mathrm{NiO} / \mathrm{Fe}_{3} \mathrm{O}_{4}$ systems $[85,86]$. Generally, the uncompensated 
moments - which may exist in a compensated interface as well — may account for most of the central EB effects (loop shift and unidirectional anisotropy). Nevertheless, exceptions were proposed; for example, a fully compensated interface can also induce a large bias as analytically suggested by Mewes and Stamps [87] assuming a distribution of anisotropy in the antiferromagnet. As a result, in addition to the surface compensation, AF anisotropies and/or interface domain structures (which can be rather critical in epitaxial samples) also factor in. Finally, it is worth noting that a strong exchange coupling can also arise in a globally compensated matrix with local uncompensated clusters exhibiting net moments. Such effect has been found in a Heusler compound with more than one element in the two sublattices contributing to the total spin magnetic moments [88]. Engineering the composition of the material on-demand for artificial compensated/uncompensated surfaces provides another alternative for tunable exchange bias in single crystallize materials.

\subsection{Spin-flop coupling}

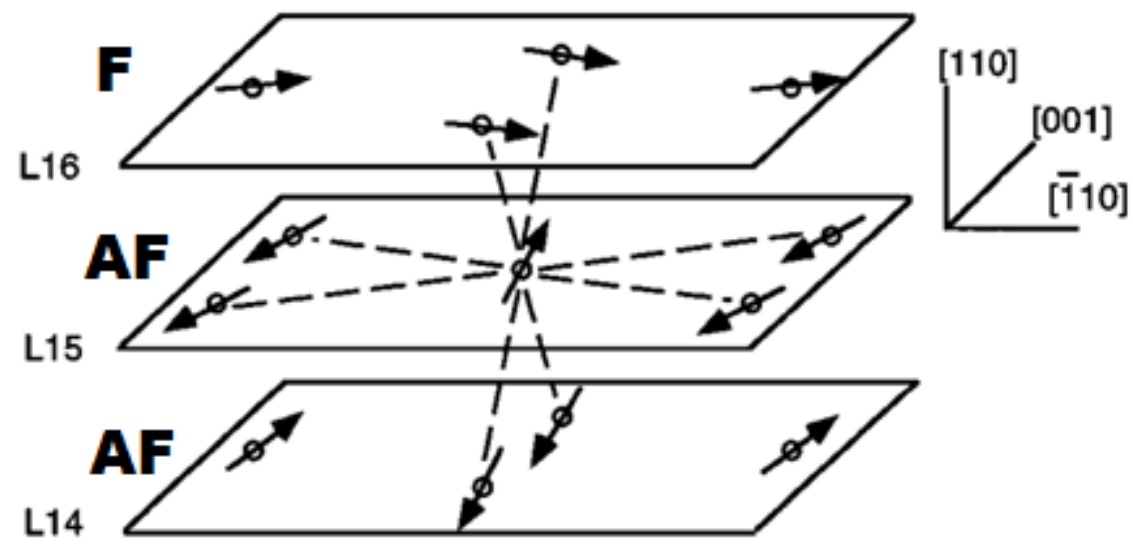

FIG. 5. (Color online) The original drawing of spin-flop coupling in exchange bias, showing the spin configuration near the interface plane for an $\mathrm{F} / \mathrm{AF}$ film with lowest energy spin orientation $\left(90^{\circ}\right)$. Reprinted from [56], copyright (1997), with permission from APS.

Spin-flop coupling refers to the perpendicular arrangement of $F$ and AF spins at the interface and is a unique type of magnetic coupling (Fig. 5), first proposed in theory [56, 89], followed by experimental confirmations of spin-flop coupling in a number of epitaxial EB systems [17, 90-96] that has led to sustained interest [90] in this subject. We focus here only on the experimental reports of spin-flop coupled systems and review their unique magnetic properties. Intuitively, spin-flop coupling arises from the long range ordering of a compensated interface, since an 
orthogonal $\mathrm{F} / \mathrm{AF}$ arrangement is a natural consequence as it minimizes the frustration of exchange coupling from the two AF sublattices; moreover, it is independent of the nature of the intrinsic F/AF coupling on the atomic scale at the interface. Phenomenologically, such coupling is described in terms of an effective uniaxial anisotropy, perpendicular to the field-cooling (FC) direction, and which allows the characterization of spin-flop coupling directly from magnetic reversal experiments, typically done by hysteresis loop and/or anisotropic magnetoresistance measurements. The uniaxial anisotropy is often recognized from an intermediate magnetization state during the magnetic reversal when measured along the FC direction. An example of a spinflop coupled Fe/MnPd bilayer is illustrated in Fig. 6. The strength of the uniaxial anisotropy can be estimated from the shift of the two sub-loops in Fig. 6(b), via $K_{\mathrm{u}}=M_{\mathrm{s}} H_{\mathrm{s}}$, where $H_{\mathrm{s}}=\left(H_{1}-\right.$ $\left.H_{2}-H_{3}+H_{4}\right) / 4$, and $M_{\mathrm{s}}$ is the saturation magnetization.
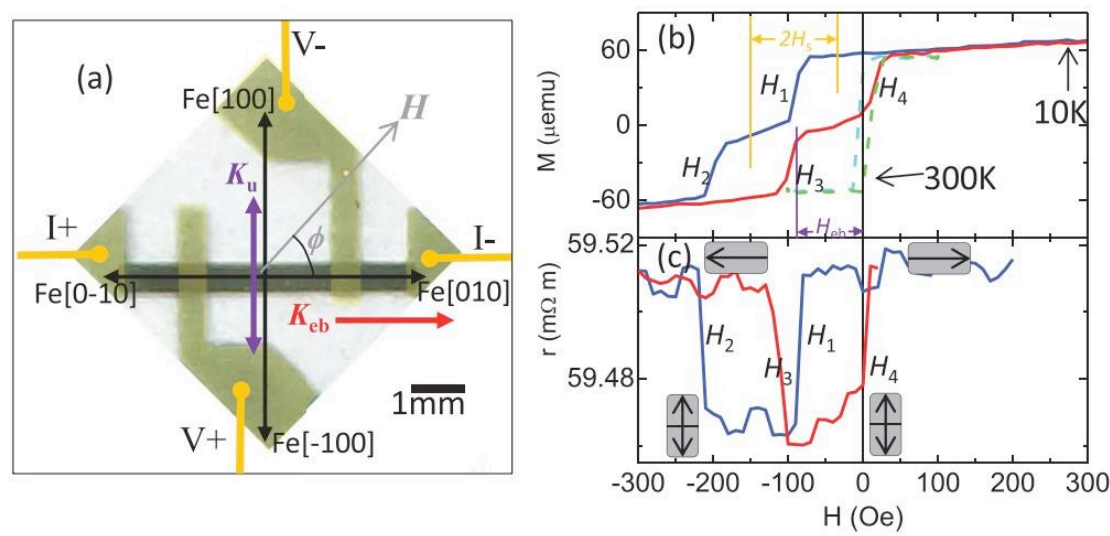

FIG. 6. (Color online) (a) Photograph of epitaxial Fe/MnPd sample (center stripe) and electrical contacts for AMR measurement. The relative orientations of the magnetic anisotropies, external field $H$, and the applied current $I$ are indicated. (b) $M(H)$ curves measured along [010] at $300 \mathrm{~K}$ (dashed) and $10 \mathrm{~K}$ (solid) after FC from 300 $\mathrm{K}$ at $H_{\mathrm{FC}}=2 \mathrm{kOe}$. (c) Corresponding $r(H)$ curve measured along [010] at $10 \mathrm{~K}$ after the same cooling process. The orientations of the Fe spins are represented by arrows enclosed in a rectangle. Reprinted from [94], copyright (2013), with permission from APS.

The spin-flop coupling alone cannot give rise to the observed loop shift. The uniaxial anisotropy from the spin-flop coupling should only enhance the coercivity of the system. If that is the case, the loop shift observed in these systems should be attributed to local, short-range interface effects instead of the long-range orthogonal coupling. Random interface roughness for finite size domains would naturally lead to a unidirectional anisotropy, according to the well-accepted Malozemoff theory [97]. In addition, practical defects such as dislocations, grain boundaries, and surface terraces, could also locally induce uncompensated regions that are oriented preferentially in the presence of the cooling field and thus break the symmetry [98, 99]. In epitaxial samples, such long-range (spin-flop) and short-range (EB) effects can sometimes be distinguished even 
from simple magnetization reversal curves, by monitoring the sharp steps (spin-flop) and the shift (EB) in the hysteresis loop, respectively. The independent origin of unidirectional anisotropy (EB) has been supported experimentally by the simple fact that realistic negative (Fe/MnPd) [92], positive $\left(\mathrm{Fe} / \mathrm{FeF}_{2}\right)$ [91], and zero-bias [100] have all been observed in spin-flop systems. The different signs of the bias can be attributed to local uncompensated spins plus the nature of F/AF coupling at the interface, i.e. either ferromagnetic (negative bias) or antiferromagnetic (positive bias) coupling. Further support for the independent origin of EB and spin-flop coupling is their different onset temperature and temperature behaviors, which have been discovered in Fe/MnPd bilayers [94], see Fig. 7(a). Another unique property of spin-flop coupling is the observed thermal hysteresis, in which the magnetization switching, from a so-called reoriented state (RS) to another aligned state (AS) and vice versa, is driven by temperature increase/decrease, under competing magnetic energies [101]. Such thermally driven hysteresis is the result of manipulating the F magnetization via competing energy terms from different origins, i.e. interface, external field and/or magnetocrystalline anisotropy. Specifically, in the reoriented state, the magnetization is aligned perpendicular to the cooling field, and is stabilized by the spin-flop coupling. Additional energy terms, such as external Zeeman energy and interface exchange energy, may favor the magnetization to align back along the FC direction. Experiments have revealed a complex phase diagram, in which four different types of thermal hysteresis have been observed (Fig. 7(b)).
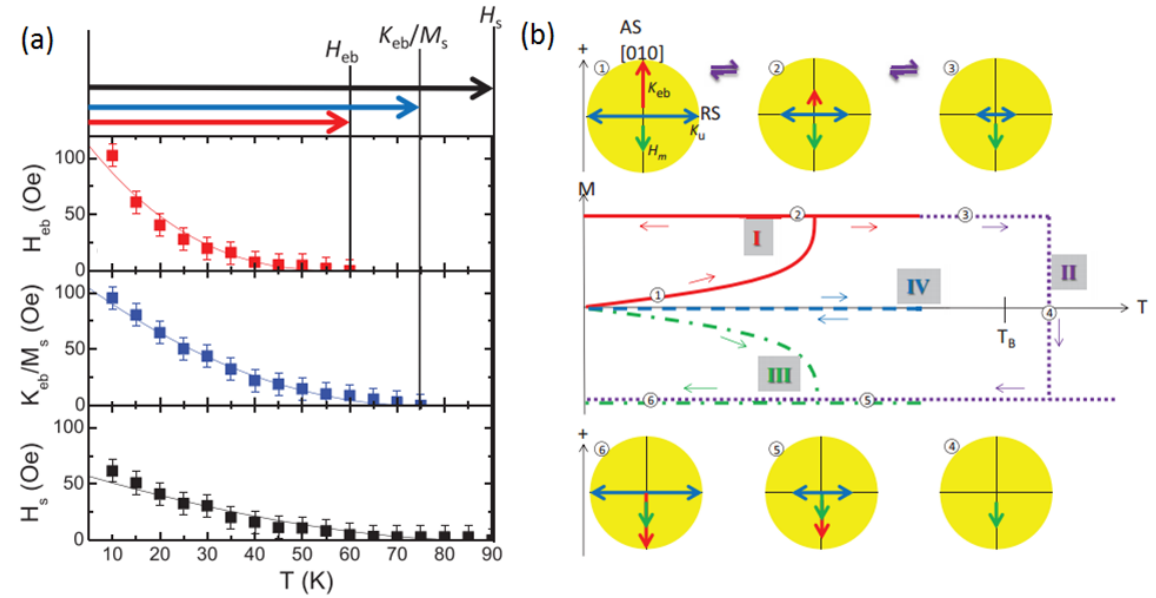

FIG. 7. (Color online) (a) Temperature dependence (symbol) and corresponding fitting (curve) by the Malozemoff model of loop shift, $H_{\mathrm{eb}}$, unidirectional anisotropy, $K_{\mathrm{eb}} / M_{\mathrm{s}}$, and uniaxial anisotropy, $K_{\mathrm{u}}\left(H_{\mathrm{s}}\right)$, showing their different onset temperatures. (b) Illustration of the temperature-driven magnetic reversals and the different types of thermal behavior in Fe/MnPd bilayers. Starting from RS, the magnetization reorients to [010] (typeI) or [0-10] (type-III) for a dominant exchange-bias $K_{\mathrm{eb}}$ or external mediating field $H_{\mathrm{m}}$, respectively. Since $K_{\mathrm{eb}}$ is temperature dependent, it is possible that the reversal is first governed by $K_{\mathrm{eb}}$ at low temperatures but then taken over by $H_{\mathrm{m}}$ at high temperatures (type-II). If $K_{\mathrm{eb}}$ and $H_{\mathrm{m}}$ are compensated, the system is dominated by the single $K_{\mathrm{u}}$; therefore, no reversal occurs throughout the temperature sweep (type-IV). The relative anisotropy strengths at selective temperatures (1-6) are also illustrated. Reprinted from [94], copyright (2013), with permission from APS. 
It is worth noting that the spin-flop coupling can be also manipulated by externally-induced anisotropy such as the shape anisotropy from lithographical structures. For example, Folven et al [102] showed that in oxide EB systems, the shape anisotropy can suppress the spin-flop coupling and even alter it into a collinear scenario (Fig. 8). This is because in nanostructures, whose physical dimensions are smaller than certain critical length scales, the edge-induced stabilization of the AF domains can overwhelm the interface coupling, resulting in a collinear alignment of the $\mathrm{AF}$ and $\mathrm{F}$ spins. Finally, certain oxide EB systems also allow convenient electric-modulation of the EB effects (sign and magnitude) via magnetoelectric couplings, which is a unique advantage in terms of integration with conventional electronics devices [103, 104].

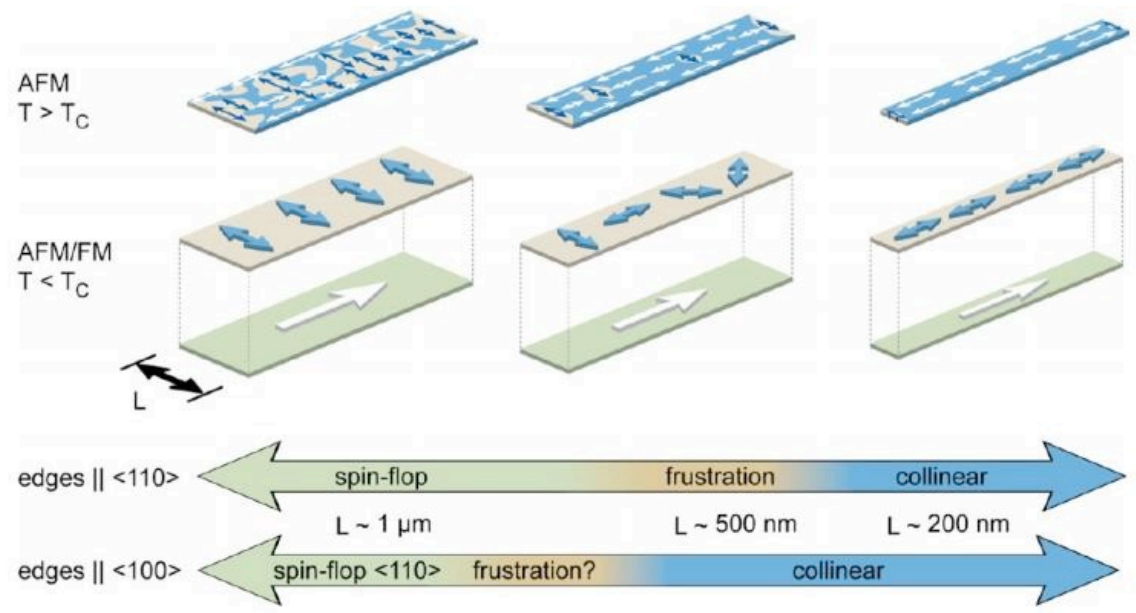

FIG. 8. (Color online) Schematic summarizing the domain configurations and spin alignment observed above and below $T_{\mathrm{C}}$ for embedded nanostructures in $\mathrm{LaFeO}_{3} / \mathrm{La}_{0.7} \mathrm{Sr}_{0.3} \mathrm{MnO}_{3}$ bilayers. Reprinted from [102], copyright (2012), with permission from ACS.

\subsection{Antiferromagnet Crystallinity}

Films with polycrystalline AF layers normally establish EB exactly along the cooling field direction. In epitaxial samples, the details of EB are determined not only by field cooling but also by the intrinsic AF crystallinity [105], especially for those AFs with strong magnetocrystalline anisotropy, such as $\mathrm{CoO}$ and $\mathrm{FeF}_{2}$ [106]. Despite the cooling field, the large AF anisotropy forces the interfacial AF spins to align with their intrinsic spin axes. For epitaxial films with only one spin axis, all interfacial AF spins point along such axis and change directions only when the cooling field is rotated far enough. An extreme example is the observation of zero exchange bias along the field cooling direction even for a well uncompensated interface, i.e. single-crystal $\mathrm{FeF}_{2}(001)$ or $\mathrm{FeF}_{2}(100)$ [36]. On the other hand, perpendicular coupling observed for a compensated $\mathrm{FeF}_{2}(110)$ interface also produces a rather small exchange bias, implying a hindered spin canting due to the large AF anisotropy. For a twinned AF with two orthogonal AF spin axes, 
the resultant exchange field is the average of the two individual exchange fields; hence it preferably lies along the bisector of the spin axes. Field cooling at various angles can only result in the exchange field rotating from one bisector direction to the other perpendicular one [107]. Quantitatively, the exchange bias is proportional to the sum of $\left|\mathrm{S}_{\mathrm{F}} \cdot \mathrm{S}_{\mathrm{AF}}\right|$ of all domains [100], where $\mathrm{S}_{\mathrm{F}}$ and $\mathrm{S}_{\mathrm{AF}}$ are the spin magnetic moments of $\mathrm{F}$ and $\mathrm{AF}$, respectively. The EB is initialized by field-cooling in polycrystalline samples, but strongly influenced by domain twinning in textured samples, and by spin canting in single-crystal samples, all depending on the AF anisotropy. For strong perpendicular $\mathrm{AF} / \mathrm{F}$ coupling, $H_{\mathrm{eb}}=0$, due to $\left|\mathrm{S}_{\mathrm{F}} \cdot \mathrm{S}_{\mathrm{AF}}\right|=0$. On the other hand, considerably larger $H_{\mathrm{eb}}$ was observed in twinned $\mathrm{Fe} / \mathrm{FeF}_{2}$ samples due to the effectively larger $\mid \mathrm{S}_{\mathrm{F}}$ $\cdot \mathrm{S}_{\mathrm{AF}} \mid, \sim 2$, when compared to uniaxial cases, $0<\left|\mathrm{SF}_{\mathrm{F}} \cdot \mathrm{S}_{\mathrm{AF}}\right|<1$ [100]. Further, it should be noted that the AF spins might undergo a spin-flop transition under a significantly stronger cooling field that exceeds the critical transition field. In such a scenario, the cooling field overcomes the AF anisotropy and alters the exchange bias (usually decreases) [108]. In addition to well-studied inplane EB systems, the effect of AF anisotropy has also been observed in out-of-plane systems such as $\mathrm{Co} / \mathrm{Pt}$ multilayers coupled with $\mathrm{CoO}$. By field cooling along different geometries, Maat et al [109] revealed that the interface exchange energies between AF and F were about twice as big for the in-plane geometry, as compared to the out-of-plane field-cooling geometry, due to the intrinsic spin axis from the $\mathrm{CoO}$ anisotropy.

The AF crystallinity also affects the behavior of the ferromagnet, through exchange coupling, by inducing and/or redistributing the spin axes (anisotropy) for magnetization reversal or changing the relevant reversal mechanisms (rotation, fanning, or domain wall (DW) movement) [100, 110115]. One of its signature consequences is the observation of asymmetric magnetization reversal [116], where the reversal mechanism is found to be different for the descending and ascending branches of the hysteresis loop. It was first discovered in $\mathrm{Fe} /$ twinned-FeF $2\left(\right.$ or $\left.\mathrm{MnF}_{2}\right)$, and later on in other EB systems as well, including Fe/MnPd [61] and $\mathrm{Co} / \mathrm{CoO}[117,118]$. Such asymmetric reversal can be also accompanied by other exotic observations including a kinked hysteresis loop [112] and incomplete domain walls [114, 119, 120]. 
a)

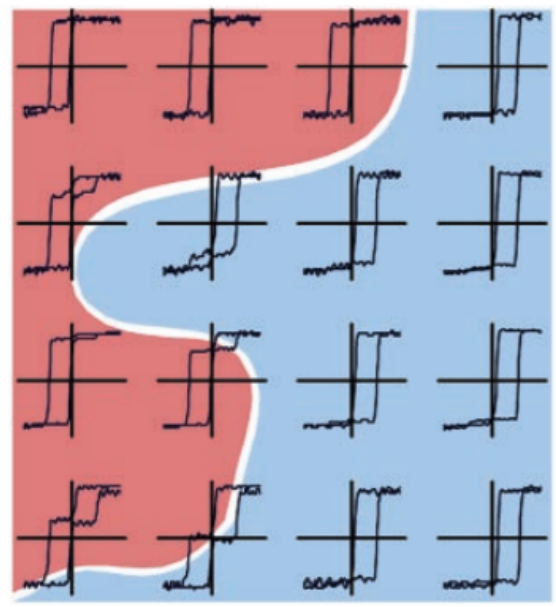

b)

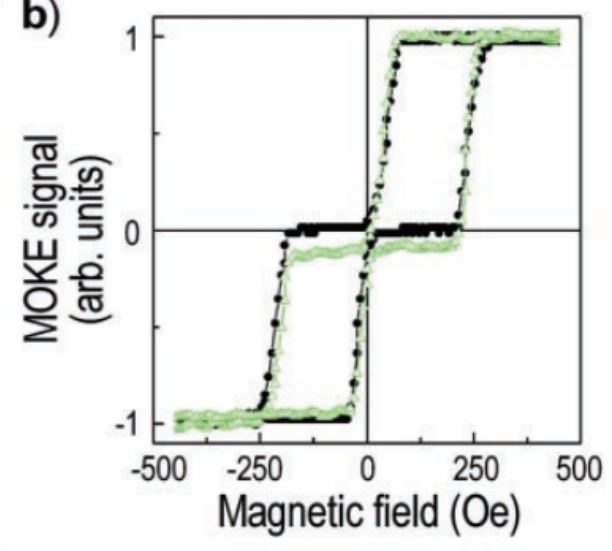

FIG. 9. (Color online) Magnetic hysteresis loops along the easy axis of an $\mathrm{FeF}_{2}(70 \mathrm{~nm}) / \mathrm{Ni}(70 \mathrm{~nm})$ bilayer, measured with MOKE on a $5 \mathrm{~mm} \times 5 \mathrm{~mm}$ sample: (a) at different parts of the sample as indicated in the figure, with a $~ 500 \mu \mathrm{m}$ laser spot; (b) from the entire sample surface area (black circles), and the average of the 16 curves in (a) (green triangles). The background color in (a) represents the local direction of the EB: red, negative; blue, positive. Reprinted from [123], copyright (2005), with permission from IOP Publishing.

The influence of AF crystallinity on the formation and lateral extent of AF domains is not easy to detect from magnetic reversal measurements. However, synchrotron and neutron techniques revealed that the antiferromagnetic domains are responsible for a number of observations including the frozen-in spins, training effects, and changes in blocking temperature [15, 121, 122]. In epitaxial samples, the AF domain size usually appears to be rather large compared to its polycrystalline counterparts (unless constrained by crystallographic imperfections such as lowangle grain boundaries or twinning). As a result, the lateral length scale of the AF domains plays a critical role in epitaxial EB systems; one consequence is the spatially non-averaging EB effect, which is established when the AF domain size is comparable or larger than the F domain size [123], see Fig. 9. Double hysteresis loops [124] can be observed due to an AF bidomain state $[125,126]$, having opposite signs (positive-EB and negative-EB) but with equal magnitude of bias acting on the F simultaneously. Furthermore, lateral and in-depth microscopic domain structures [127] in the F can be also imprinted by the opposite AF domain structures.

\subsection{Surface terminating layers}

Epitaxial structures are more susceptible to local defects, such as roughness [128], inter-diffusion [129], and terminating layers. Different atomic-step-induced terminating layers (integral or halfintegral monolayer) for epitaxial F/AF interfaces are summarized in Fig. 10. For bimetallic AFs such as $\mathrm{MnPd}$, a compensated interface is still compensated whenever the surface ends on integral 
or half-integral monolayer. However, an uncompensated interface may become locally compensated due to the formation of a half-integral monolayer. A similar scenario can be found in single-element metallic AFs such as Mn. The compensated, in-plane, $c(2 \times 2)$ reconstructed Mn

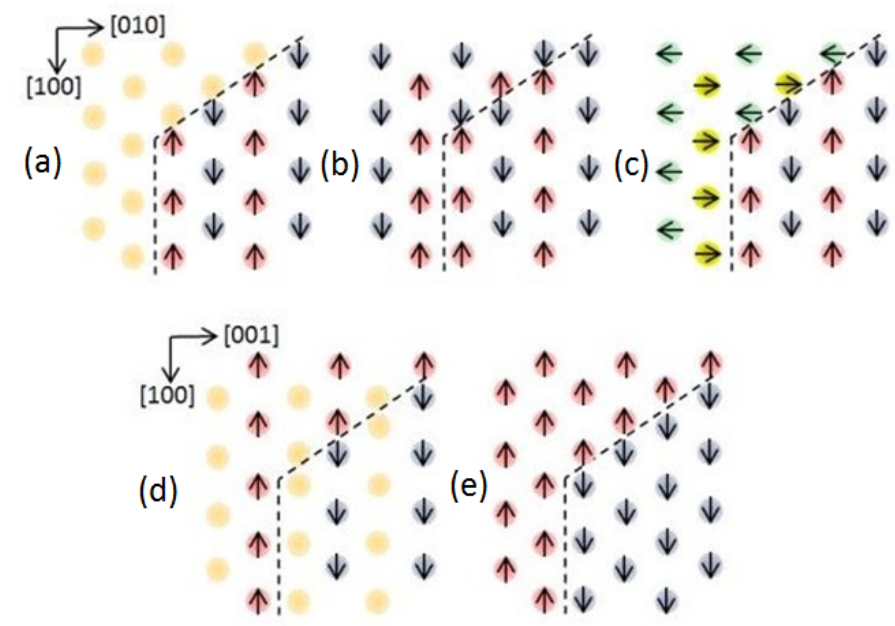

FIG. 10. (Color online) Schematic summarizing the modification of the surface compensation, coupling and spin orientation by different atomic terminating layer(s) in epitaxial EB systems. Half-integral monolayer induced changes for antiferromagnetic, compensated surfaces: (a) with and (b) without the loss of surface spin magnetic moments, and (c) with changing orientations of the spin magnetic moments (changing the effective coupling mechanism). The surface compensation is not affected for the above cases. (d) and (e): Similar half-integral monolayer induced effects for uncompensated surfaces. The nominally uncompensated surfaces can be broken and exhibit local compensation.

phase remains compensated for a half-integral monolayer, and constantly exhibits a spin-flop F/AF coupling. However, the uncompensated AF phase may be locally compensated by halfintegral monolayer, thus changing the effective coupling type from collinear to spin-flop [78]. For epitaxial FeMn(001) and along certain orientations, the AF spins are perpendicularly arranged for each adjacent layer. The half-integral monolayer, in the form of atomically raised islands, can result in biaxial coupling and thus an induced four-fold anisotropy [79].

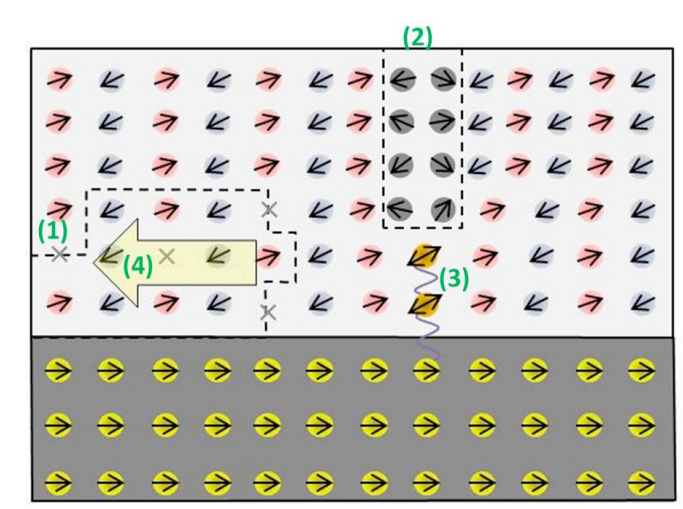

FIG. 11. (Color online) Schematic summarizing the effects of defects and nonmagnetic impurities (1), dislocations (2), rotatable spins (3), and uncompensated moments (4) on the exchange bias properties. 
Effects of nonmagnetic impurities at the F/AF interface and/or in the bulk of the AF have induced much discussion in relation to their contributions to the AF domain formation [98, 130-133]. The exchange field can be enhanced due to increased density of uncompensated magnetization in AF domains, whose formation can be made easier by the fact that the DWs passing through those interfacial defects are energetically favorable. On the other hand, further increasing the defects, may either hinder the domain formation or reduce the net uncompensated spins, both are unfavorable for EB. In the AF bulk, the strained AF layers, buried under the interface, can relax to the bulk lattice structure and lead to the formation of dislocations as the film grows thicker. Such relaxation behavior is obvious even from simple XRD experiments [92] in epitaxial samples. Frustrated spins exist under these dislocations and possess weaker coupling to their neighboring spins as compared to perfect crystals. During the magnetization reversal, these frustrated spins [134] become easily rotatable under strong F/AF coupling and weak AF anisotropy (Fig. 11). Such a scenario is used to explain the dramatic enhancement of the rotatable AF components by the presence of increased numbers of dislocations in epitaxial EB bilayers with low AF anisotropies [135].

\subsection{Antiferromagnet bulk effects}

The larger crystallographic coherence lengths in epitaxial samples usually gives rise to longer spin correlation length compared to polycrystalline samples. The domain size for epitaxial $\mathrm{FeF}_{2}$ is $\sim 500 \mathrm{~nm}$ [136], for $\mathrm{NiO}$ is $150 \mathrm{~nm}$ [137], and for epitaxial FeMn is $\sim 50 \mathrm{~nm}$ [138], almost double of what is observed in polycrystalline samples. As a result, AF spins buried deeply in the AF bulk still contribute to the EB effect [139]. F/AF/F sandwich structures are often used to study this bulk effect by monitoring simultaneously the magnetization reversals of the two F layers [140]. Long-range interlayer coupling and propagation of EB were first studied in trilayer samples with a FeMn layer sandwiched by two different F layers (CoFe or FeNi) [138, 140]. Bulk effects with collinear interface coupling was investigated in $\mathrm{Ni} / \mathrm{FeF}_{2} / \mathrm{NiFe}$ sandwich [139] and with perpendicular interface coupling in epitaxial Fe/MnPd/Fe/IrMn multilayers [67], with an additional IrMn pinning layer on top of the sandwich. In the latter multilayer structure, the IrMn $\left(T_{\mathrm{B}}>\mathrm{RT}\right.$ ) layer is used to provide a strong pinning of the second Fe layer adjacent to it, which makes the magnetic reversal of the two Fe layers occurs at different field strength. On the other hand, due to the low blocking temperature of $\operatorname{MnPd}\left(T_{\mathrm{B}} \sim 90 \mathrm{~K}\right)$, its bulk spin arrangements can 
be defined by cooling the samples from above to below the $T_{\mathrm{B}}$ of $\mathrm{MnPd}$ under different arrangements of the two Fe layers, i.e. either in parallel or antiparallel configurations.

Observations of how the second Fe magnetization history in the Fe/IrMn couple affects the EB of the $\mathrm{Fe} / \mathrm{MnPd}$ couple provide direct evidence for the specific role played by the bulk MnPd spins. In Fig. 12, the different switching fields observed in the minor loop (reversing only the first Fe layer) and major loop (reversing both Fe layers) indicates that a non-recoverable change in the MnPd can occur during second Fe magnetization switching, i.e. the bulk MnPd spin structure is affected by the second Fe magnetization configuration.
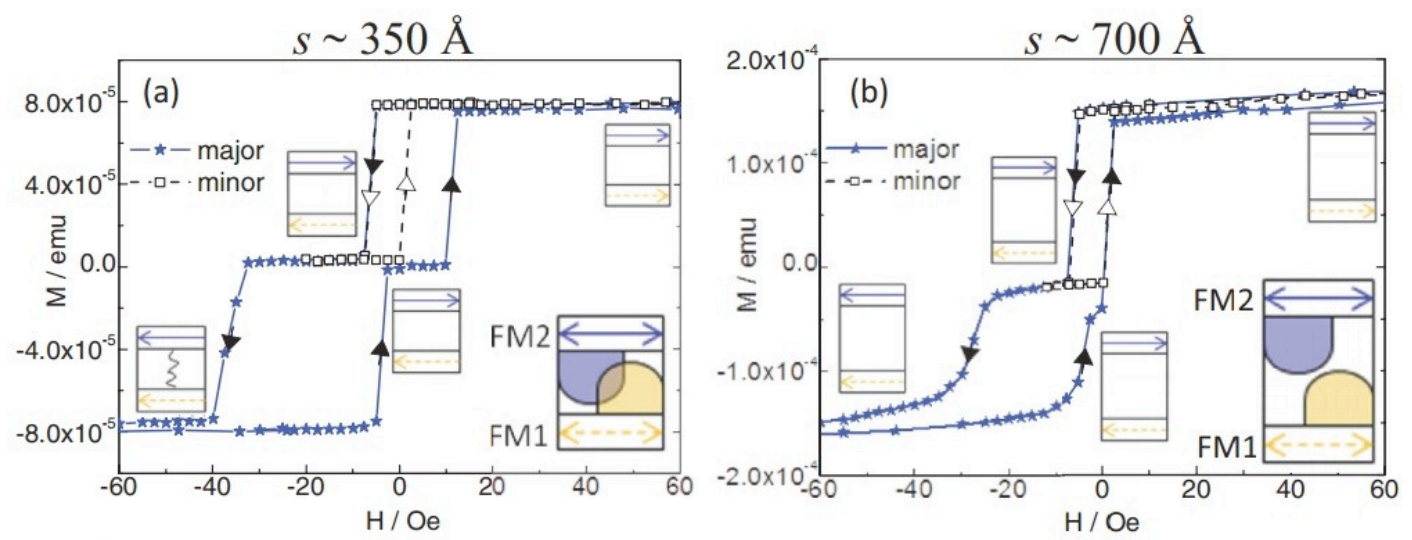

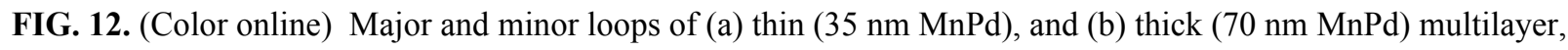
samples with the structure of $\mathrm{Fe} / \mathrm{MnPd} / \mathrm{Fe} / \mathrm{IrMn}$ at RT. Magnetization configuration of the first $\mathrm{Fe}$ (FM1, dashed arrow) and the second Fe (FM2, solid arrow, pinned by IrMn) are illustrated in an enclosed box for each magnetic switching. Reprinted from [67], copyright (2012), with permission from APS.

Reciprocally, the depth of the AF bulk spins that can be affected during the F magnetization reversal can also be reflected from the critical thickness at which the EB saturates [67, 94, 141], as shown in Fig. 13. We note that large saturation thicknesses are also found in epitaxial EB samples. For example, the saturation thickness in epitaxial $c$-axis Fe/MnPd bilayers is experimentally determined to be $>80 \mathrm{~nm}$ (Fig. 13). The DW nucleation energy and the induced uniaxial anisotropy also increase with the MnPd thickness [135]. Such length scales are usually on the order of several nanometers for polycrystalline samples. 


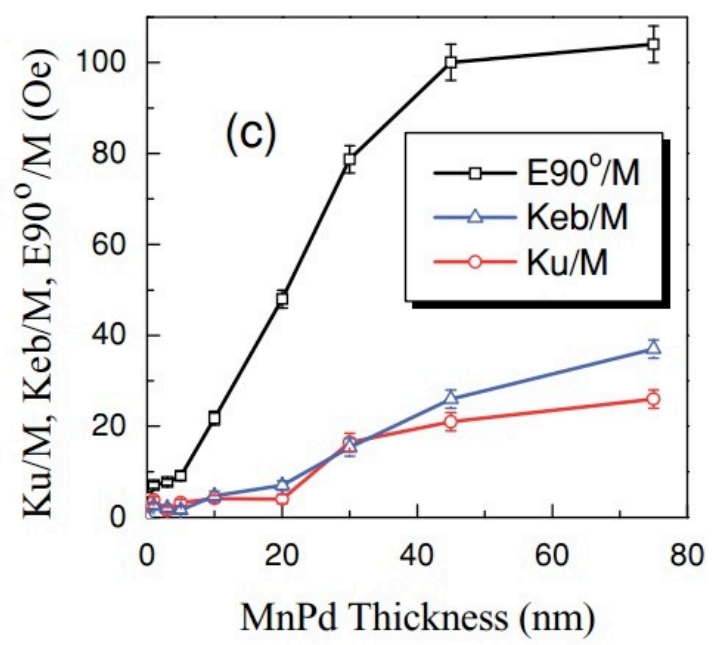

FIG. 13. (Color online) Thickness-dependence of EB and induced magnetic anisotropies in $c$-axis normal $\mathrm{MnPd} / \mathrm{Fe}$ bilayers, showing the non-saturation effect up to $80 \mathrm{~nm}$ of MnPd thickness. Reprinted from [135], copyright (2011), with permission from APS.

Other important physical length scales for AFs, such as the AF domain size and AF correlation length, have also been studied in nanostructures (e.g. nanodots and nanowires) [142] whose size and dimension are artificially made comparable to these physical length scales. In the case of larger dot sizes that exceed the AF domain sizes, the EB effect originates mainly from modified ferromagnetic behaviors, i.e change in the demagnetizing energy of the nanodots compared to other important energies in the system (Zeeman, exchange, and anisotropy) [143]. For comparable dot- and domain-sizes, opposite results have been reported: Baltz et al. [144] found that EB weakly depends on the AF thickness in nanostructures due to the fact that the lateral dimensions of the dots physically limit the AF domain size. A reduced blocking temperature was also found which is not unexpected for the nanostructures due to the finite-size effect. Liu et al [145] reported enhanced EB due to changing the magnetization reversal mechanisms of the nanodots. In particular, coherent rotation is favored over DW movements in the nanodots. Malinowski et al [146] reported enhanced EB in an out-of-plane system that is due to the lateral confinement of the AF domains. Similar EB enhancement was also observed by Laureti et al [147], but the mechanism was attributed to the increased interface coupling energy that showed an inverse relationship with the AF correlation length. In contrast, there are some literature reports of a decreased EB field in nanostructured samples when compared with their thin-film counterparts. Increased magnetostatic interaction (demagnetization) [148, 149], and constrained/destabilized AF domains $[150,151]$, are considered to be the main sources for such reduction. It is believed that the $\mathrm{EB}$ effects in nanostructures are closely related to the AF domain formation. In epitaxial samples with large intrinsic AF domains, the nanostructures define an 
upper limit of the domain size (promote the domain formation) and thus, in general, enhance the EB effects; only when the intrinsic domains are small, the EB may be reduced through other dimension-sensitive parameters, such as destabilization of AF domains, random (frustrated) edges [152], or incomplete F/AF coupling [136].

\section{Competing Anisotropies}

Magnetic reversal is determined largely by magnetic anisotropies. EB is viewed as a unidirectional anisotropy that pins the magnetization along one specific direction. In the samples with epitaxial AF layers, the optimized structural coherence and well-defined magnetic ordering induce additional magnetic anisotropies, which result in multiple, competing spin easy axes with distinct symmetries. Additionally, the magnetocrystalline anisotropy of the F layer also needs to be considered if the $\mathrm{F}$ layer is also epitaxially grown. Different types of magnetic reversal coherent rotation and domain wall movements - have been found for different epitaxial systems with competing anisotropies. In the second part of this review, we provide a summary of the role of magnetic anisotropies in EB systems and their effects on the magnetic reversal behavior. These anisotropies have different origins: (1) interface-induced exchange anisotropy (unidirectional); (2) AF-induced anisotropies (uniaxial, 3-fold, 4-fold, 6-fold); (3) ferromagnetic anisotropy (uniaxial, cubic); (4) extrinsically induced shape anisotropy. Finally, we introduce phenomenological models that offer a quantitative connection between the magnetic reversal properties and the competing anisotropies.

\subsection{Unidirectional anisotropy}

Exchange bias manifests itself as a unidirectional anisotropy that is influenced by various parameters such as materials, thickness, temperature, field-cooling, defects, interface, ferromagnetic state, and training effect $[2-5,7]$. To briefly summarize, studies of the effects of AF layer thickness showed that such anisotropy is establishes at a certain minimum thickness, usually on the order of several $\mathrm{nm}$, and reaches saturation at a larger thickness, on the order of several tens of $\mathrm{nm}[153,154]$, and thus the exchange anisotropy increases and then saturates at a critical value with thickness. However, if there is the possibility of net AF moments (from uncompensated spins) at the interface [155-157], a slightly complicated, nonlinear AF-thickness dependence [155] which increases first, goes through a maximum before saturation, is observed and supported by an analytical model [156]; for example in Co/IrMn systems [154]. 
Temperature dependence of EB has been interpreted by various theoretical approaches [158-163] in which AF domains and interfacial disorders are believed the key parameters dictating the dependences. Field-cooling is a decisive control for EB; however, as we have discussed earlier, it needs to compete with the intrinsic AF spin axes in epitaxial systems. Misaligned unidirectional anisotropy can be caused by non-collinear field-cooling [164] or interfacial frustration [165]. Non-averaged field-cooling effect can result in the coexistence of positive and negative exchange bias and unique maze-like domain patterns. It is also noted that in EB bilayers with strong ferromagnetic anisotropy, the remnant ferromagnetic state alone is sufficient to pin the magnetization and establish the unidirectional anisotropy upon cooling, thus no external field is even necessary [166].

Defects, although hard to tackle due to their microscopic scales, have direct influence on the interface unidirectional anisotropy. A series of theoretical papers by Kim and Stamps [167-169], address defect-mediated reversal in which the magnetization reversal was described to be controlled by an attractive domain-wall potential in the AF bulk arising from magnetic impurities whose locations relative to the interface are responsible for the magnitude of both EB and coercivity. Finally, a number of exotic properties, including rotation $[170,171]$ and sign-reversal [134] of the unidirectional anisotropy, have been correlated to training effects. Specifically, changes of the interface AF domain pattern during the cycling of the magnetization reversal (training) account for the above observations [52, 172-175].

\subsection{Two-fold (uniaxial) anisotropy}

Interface-induced uniaxial anisotropy is commonly observed in F/AF epitaxial EB systems. It is attributed, at least partly, to the rotatable AF spins at the interface. The magnitude of such anisotropy depends on the strength of the intrinsic AF anisotropy and the exchange coupling. For an EB interface with strong exchange coupling and low AF anisotropy, the uniaxial anisotropy is usually quite large. In polycrystalline F/AF bilayers, such uniaxial anisotropy can be estimated by the enhanced coercivity compared to that of a single F layer. Such anisotropy is collinear with EB in the collinear coupled F/AF systems, but is perpendicular with respect to the EB in spin-flopcoupled F/AF systems.

Competing exchange, $K_{\mathrm{eb}}$, and uniaxial, $K_{\mathrm{u}}$, anisotropies have been studied as a function of their relative magnitudes, symmetry, temperature dependence, and orientation. One interesting effect is 
the $\mathrm{Keb}_{\mathrm{eb}} / \mathrm{K}_{\mathrm{u}}($ ratio)-dependent reversal asymmetry. The magnetic reversal routes are directly related to such anisotropy ratio $[176,177]$, instead of their absolute magnitudes, and we summarize some important results in this area of investigation. Camarero et al [178] modeled the angle-dependent magnetization reversal process via the Stoner-Wohlfarth (SW) approach, taking into account the competing $K_{\mathrm{eb}}$ and $K_{\mathrm{u}}$ values. Beckmann et al [179], via Monte-Carlo simulations, found that the anisotropies directly correlate with the different magnetization reversal modes, either coherent rotation or DW movements. Hoffmann [180] studied the symmetry of the anisotropies and proposed an analytical model that directly explains the training effect. Grimsditch et al [181] studied the relationship between anisotropies and the temperature-dependent exchange bias and coercivity. Jimenez et al investigated the effect of the misalignment of $K_{\mathrm{eb}}$ and $K_{\mathrm{u}}$ on the magnetization reversal, in which the misalignment was promoted either by field cooling or interfacial frustration $[164,165]$. These results were later confirmed with direct imaging by McCord et al [182]. Finally, in epitaxial samples with strong magnetocrystalline anisotropy, the uniaxial anisotropy can also break the overall symmetry of the magnetocrystalline anisotropy and induce asymmetrical magnetization reversal [183, 184].

\subsection{Three-fold anisotropy}

To our knowledge, only one experiment described a three-fold induced anisotropy due to exchange bias. By using AMR measurement on $\mathrm{Fe} / \mathrm{MnF}_{2}$ bilayers, a unique three-fold anisotropy was revealed in addition to the biaxial, unidirectional, and uniaxial components. The three-fold component has a magnitude similar to the uniaxial and biaxial components, of the order of 0.01 $\mathrm{erg} / \mathrm{cm}^{3}$, and was found to be responsible for the symmetry-breaking of the magnetization reversal process [185]. Last but not least, it is theoretically proposed that the triangular lattice can also give rise to a strong three-fold anisotropy, such as in $\operatorname{IrMn}_{3}[186]$.

\subsection{Four-fold (cubic) anisotropy}

Induced four-fold anisotropy has been observed in $\mathrm{Co} / \mathrm{FeF}_{2}(110)$ [181], $\mathrm{Fe} / \mathrm{MnF}_{2}(110)[113,185]$, $\mathrm{NiFe} / \alpha-\mathrm{Fe}_{2} \mathrm{O}_{3}$ [187], $\mathrm{NiFe} / \mathrm{FeMn}$ [188], and $\mathrm{FeMn} / \mathrm{Co}(001)$ systems [79] but with different physical origins. For a Co layer on a single crystalline $\mathrm{FeF}_{2}$ film, the fourfold anisotropy only appears near the Néel temperature; this was attributed to the short-range order of the antiferromagnet. For an Fe film grown on a twinned quasi-epitaxial $\mathrm{MnF}_{2}(110)$ layer, the fourfold anisotropy appears suddenly at the $\mathrm{MnF}_{2}$ Néel temperature and increases strongly with decreasing temperature. It is therefore believed to be induced by the twinned AF domains in the 
antiferromagnetic $\mathrm{MnF}_{2}$ film due to the strong coupling from the $\mathrm{Fe} / \mathrm{MnF}_{2}$ interface, so the fourfold anisotropy has the easy direction midway between the AF spin directions of $\mathrm{MnF}_{2}$ twinned domains. For $\mathrm{NiFe} / \alpha-\mathrm{Fe}_{2} \mathrm{O}_{3}$, the anisotropy comes from the frozen-in $\mathrm{AF}$ domains below $T_{\mathrm{N}}$, which is not sensitive to field annealing. Mewes et al [188] showed that the spin frustrations within a multi-sublattice AF material (such as FeMn) can lead to a four-fold anisotropy for a compensated, flat surface. Similarly, for single crystalline FeMn/Co(001) system without twinned AF structures, the spin frustrations at atomic steps of the locally compensated FeMn(001) surface is the source of the induced fourfold anisotropy. Specifically, the FeMn AF spins above the steps (along $\pm[110]$ ) and below the steps (along $\pm[1 \overline{1} 0]$ ) oriented $90^{\circ}$ with respect to each other, frustrates the FeMn/Co exchange interaction and leads to easy axes along the [100] directions, midway between the AF spin directions of the FeMn $90^{\circ}$ domains. Finally, dependence of the fourfold anisotropy on both thickness [189] and temperature [80] were reported in Fe/CoO systems.

\subsection{Six-fold anisotropy}

Six-fold anisotropy was found in epitaxial $\mathrm{NiFe}(111) / \mathrm{CoO}(111)$ by angle-dependent neutron scattering experiments. Oscillations of the peak intensities with a periodicity of $60^{\circ}$ for both nonspin-flip and spin-flip [12] neutron signals were observed, which is in good agreement with a sixfold symmetry from the structural characterization. The AF spins (domains) follow closely the anisotropy axes and are not randomly orientated in plane. These anisotropic orientations of the AF domains provide, on average, a virtually compensated interface and therefore lead to an EB field several orders of magnitude lower than expected. It is believed that although each AF domain provides locally a bias projection along the cooling direction giving a large finite bias, it averages out due to the anisotropic lateral orientations parallel to all crystallographic directions. The interfacial coupling of the local biasing only manifests itself in the hysteresis loop as a strongly enhanced coercivity [15].

\subsection{Ferromagnetic magnetocrystalline anisotropy}

The magnetocrystalline anisotropy of the ferromagnetic layer also influences magnetization reversal in epitaxial systems. In polycrystalline films, the $\mathrm{F}$ anisotropy is insignificant and the magnetic reversal behavior is largely determined by the physical microstructure. The small grains resemble small SW particles and the reversal can be modeled using the coherent rotation protocol [7]. In single crystalline films where long-range structural order is maintained, the exchange 
coupling aligns spin magnetic moments to the anisotropy easy axes [190], forming large and stable domains, and driving the magnetization reversals primarily by DW nucleation and propagation. For example, a quantitative model has been proposed to interpret the magnetization reversal of epitaxial Fe thin films by taking into account the static energy state at each anisotropy direction [191]. The interplay between magnetocrystalline and the induced uniaxial anisotropies has been systematically studied by Zhan et al. [192-194]. Magnetic switching fields were predicted by the model considering the DW nucleation energy and phenomenologically including additional induced-anisotropy terms, which usually have lower symmetries and can change the ground state energy for each axis [195]. The interplay between F magnetocrystalline anisotropy and EB has been reported in several epitaxial systems. Generally, one would expect magnetization reversal characteristics such as stepped hysteresis loop and reversal asymmetry $[183,196]$, which are attributed to the complex configuration of multiple easy axes $[32,197,198]$. Dual reversal modes and complex angular dependences [199] were found, in which the multiple reversal steps were the result of successive DW movements [200, 201].

\subsection{Extrinsically induced shape anisotropy}

Due to the recent developments in lithographic fabrication and nanopatterning, various magnetic nanostructures, including 1D nanowires and 0D nanodots [202-207], can be fabricated with tunable magnetic shape anisotropies. For example, in nanowire arrays, a large uniaxial anisotropy is induced that is significantly larger than that of the interface-induced anisotropies. Such anisotropy can directly modify the magnetic reversal characteristics. Angle dependent magnetometry measurements show that the exchange bias is a strong function of the field cooling

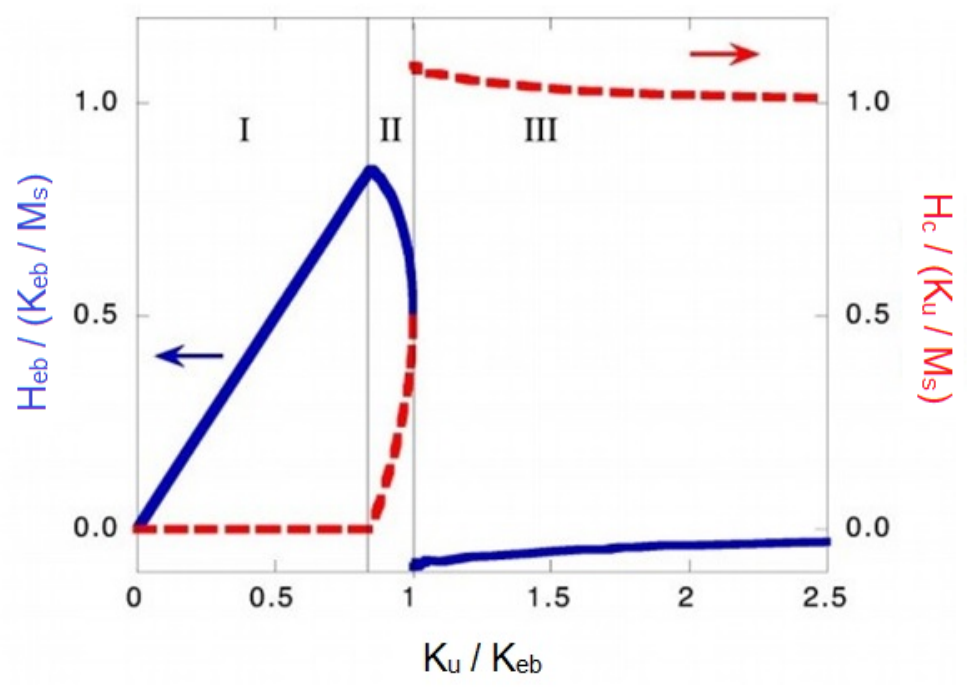


FIG. 14. (Color online) Calculated exchange bias, $H_{\mathrm{eb}}$ (solid lines), and coercivity, $H_{\mathrm{c}}$ (dashed lines), as a function of $K_{\mathrm{u}} / K_{\mathrm{eb}}$ ratio of $\mathrm{Fe}$ lines on top of a continuous $\mathrm{FeF}_{2}$ antiferromagnet. Reprinted from [177], copyright (2003), with permission from APS.

orientation with respect to the long axis of the nanowire [208]; besides, the magnetization reversal mechanism evolves from curling to coherent rotation by changing the aspect ratio of the nanowires [209]. Hoffmann et al [177] demonstrated that the uniaxial shape anisotropy induced by lithographic patterning can give rise to an additional EB field whose magnitude depends on the critical ratio, $K_{\mathrm{u}} / K_{\mathrm{eb}}$. In the $K_{\mathrm{eb}}$-dominated region (I), increasing the $K_{\mathrm{u}} / K_{\mathrm{eb}}$ ratio would rapidly enhance the measurable EB field, but contribute little to the coercivity; however, in the $K_{\mathrm{u}^{-}}$ dominated region (III), the measured coercivity is way larger than the bias field, and increasing the $K_{\mathrm{u}} / K_{\mathrm{eb}}$ ratio would actually slightly reduce the coercivity but enhance the bias. Furthermore, they observed a first-order transition close to the critical condition, $K_{\mathrm{u}} / K_{\mathrm{eb}} \sim 1$ in the $\left(H_{\mathrm{eb}}, H_{\mathrm{c}}\right)$ vs. $K_{\mathrm{u}} / K_{\mathrm{eb}}$ phase diagram (II), where both $H_{\mathrm{c}}$ and $H_{\mathrm{eb}}$ change abruptly (Fig. 14). Chung et al further showed that the loop shift in nanowires can be enhanced up to $40 \%$ larger than the value one would naively expect from the interfacial coupling alone [210].

\section{Analytical Models}

\subsection{Effective field model}

Phenomenological models are useful approaches for quantitatively understanding the magnetic reversal in epitaxial EB samples. Competing anisotropies with distinct symmetries lead to complex magnetic reversal behavior and angular dependence of EB and coercivity [211,212]. Part of the complexity arises from different and/or mixed magnetic reversal modes for different measurement conditions. Crossover of the magnetic reversal modes can occur at different external field directions and/or different temperatures. The original Meiklejohn-Bean (MB) approach to EB takes the magnetization as a macroscopic spin that coherently rotates during the magnetization reversal. In this approach, one would model the magnetization reversal by solving the energy minimum of the total Hamiltonian of the magnetic system. It has demonstrated great success in analyzing polycrystalline EB samples and is especially helpful for the interpretation of the angular dependence $[80,213]$. However, due to the simple assumption of a macrospin throughout the reversal process, such models often fail to predict the many details or particularities of the reversal behavior found in epitaxial systems, including asymmetries and intermediate states. To avoid overlooking the details during the reversal, one has to first carefully re-examine the reversal 
mechanisms, which can be potentially influenced by the long coherency induced by epitaxy as well as the exchange bias. For example, under the symmetric-breaking conditions of exchange bias, the reversal process can take qualitatively different physical mechanisms along different branches of the hysteresis loop, i.e. either coherent rotation or DW nucleation-propagation, as was directly observed by in microscopy experiments using XMCD for magnetic contrast in $c$-axis $\mathrm{MnPd} / \mathrm{Fe}$ bilayers [61]; see Fig. 15.
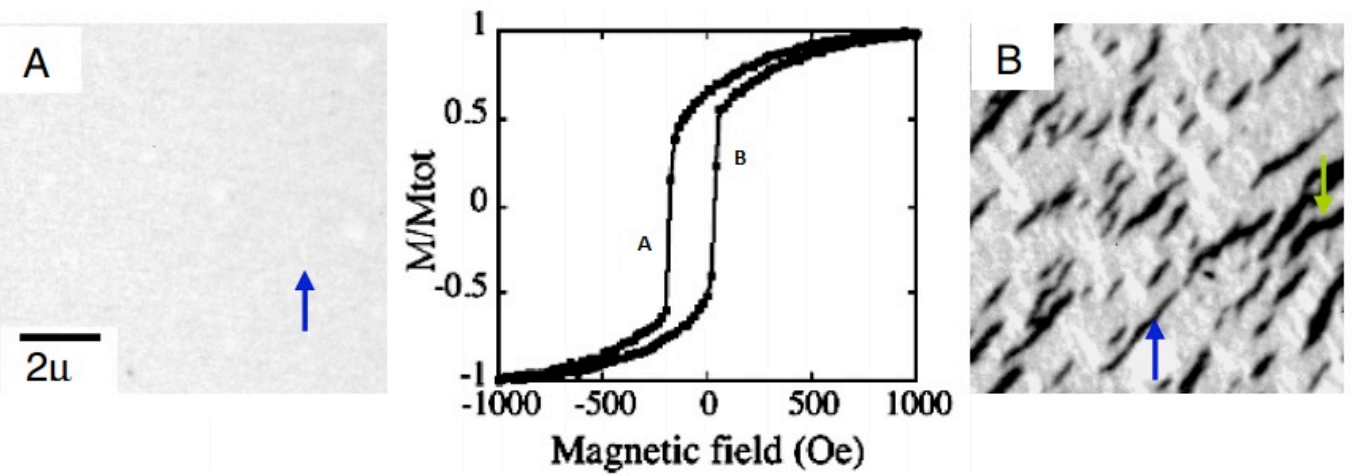

FIG. 15. (color online) Magnetic reversal of $c$-axis Fe/MnPd bilayers follows different reversal mechanisms for (A) descending branch under coherent rotation, and (B) ascending branch under DW nucleation and propagation. Reprint from [61], copyright (2005), with permission from APS.

The pioneering work by Beckmann et al [179] set the foundations for the effective-field approach for modeling exchange bias, with the advantage of extracting realistic anisotropy-field values without specifying the underlying reversal mechanisms. In order to quantitatively understand the magnetic reversal at different field orientations, the magnetization is considered to be determined

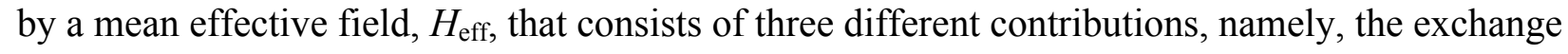
field, $H_{\mathrm{ex}}=J_{\mathrm{int}} m_{\mathrm{AF}}$, that is proportional to the interface exchange constant, $J_{\text {int }}$, and the AF net magnetization, $m_{\mathrm{AF}}$, the external magnetic field, $H_{\mathrm{Z}}$, and the anisotropy field, $H_{\mathrm{A}}$, that is proportional to the F magnetization. Arenholz et al [198] and Zhang et al [35, 214] extended this model for the interpretation of $\mathrm{Fe} / \mathrm{MnF}_{2}$ and $\mathrm{Fe} / \mathrm{IrMn}$ epitaxial systems, respectively. Effective fields on the order of several tens of Oe were obtained which matches quite well with the DW nucleation fields of epitaxial ferromagnetic films.

\subsection{Domain wall nucleation model}

\subsubsection{Description of the model}

Zhan, Zhang and Krishnan [135] further pointed out that the measured ferromagnetic anisotropy field is nothing but the $90^{\circ}$ and $180^{\circ} \mathrm{DW}$ nucleation energy, by experimentally studying the prototypical, epitaxial-(002) EB systems. This analytical approach was then 
formulated as the 'DW nucleation model', in which the DW nucleation and propagation are determined by the DW nucleation energies, namely, $\varepsilon_{90}{ }^{\circ}$ and $\varepsilon_{180^{\circ}}$, as well as other additional anisotropies induced by external origins (interface, surface morphologies, etc.) including $K_{\mathrm{eb}}$ and $K_{\mathrm{u}}$. (Fig. 16). For epitaxial-(002) systems such as Fe/MnPd, the total energy for the ferromagnetic layer can be written as [135]:

$$
E=\frac{K_{1}}{4} \sin ^{2} 2 \theta-K_{u} \cos 2 \theta-K_{e b} \cos \theta-M H \cos (\varphi-\theta)
$$

where $K_{1}$ is the ferromagnetic anisotropy, $\theta$ is the angle between the magnetization and the bias direction, and $\phi$ is the angle between the applied field, $H$, and $K_{e b}$.

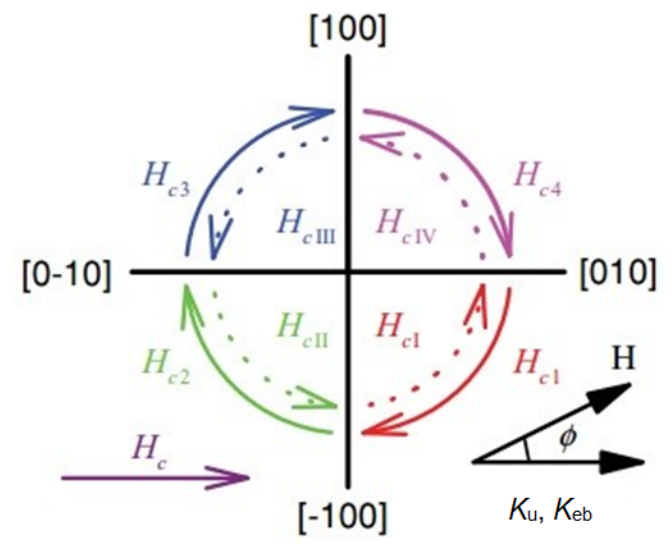

FIG. 16. (Color online) The anisotropy geometry for epitaxial Fe/MnPd EB bilayers. Fe cubic anisotropy is superimposed on the EB-induced unidirectional, $K_{e b}$, and uniaxial, $K_{u}$, anisotropies aligned along the $\mathrm{Fe}[010]$ axis. The coercivities, $H_{c l}-H_{c 4}$ and $H_{c l} H_{c l V}$, for the magnetic switching between different $\mathrm{Fe}$ easy axes are defined. The external field is applied at an angle $\varphi$ with respect to the bias direction. Reprinted from [135], copyright (2011), with permission from APS.

Instead of modeling the hysteresis by solving the above Hamiltonian, only the distinct energies of single domain states at the four Fe easy axes orientations are considered, and these axes are assumed to be the only (meta)-stable states for the ferromagnetic spins. Therefore, the switching fields are determined by the nucleation energies of the specific types of DWs, which can be derived from the energy gained between the local minima at the initial and final easy axes involved in any possible magnetic transitions (Fig. 16). Additional anisotropies, such as $K_{\mathrm{eb}}$ and $K_{\mathrm{u}}$, further break the symmetry causing the energies of single domain states, originally aligned along the degenerate easy-axes orientations, to evolve differently. As a result, the switching fields between the different easy axes can be accurately predicted, which are functions of the nucleation energies, $\varepsilon_{90}{ }^{\circ}$ and $\varepsilon_{180}{ }^{\circ}$, anisotropy constants, $K_{\mathrm{eb}}$ and $K_{\mathrm{u}}$, and the external field [135]. Figure 17 show typical example of the evolution of the angular-dependent switching fields (symbols) of epitaxial $\mathrm{Fe} / \mathrm{MnPd}$ fitted by the DW nucleation model (curves). Both one-step and two-step switching, 
observed in experiments (using MOKE [135, 214] and/or AMR [215]), can be nicely modeled within the whole angular range.

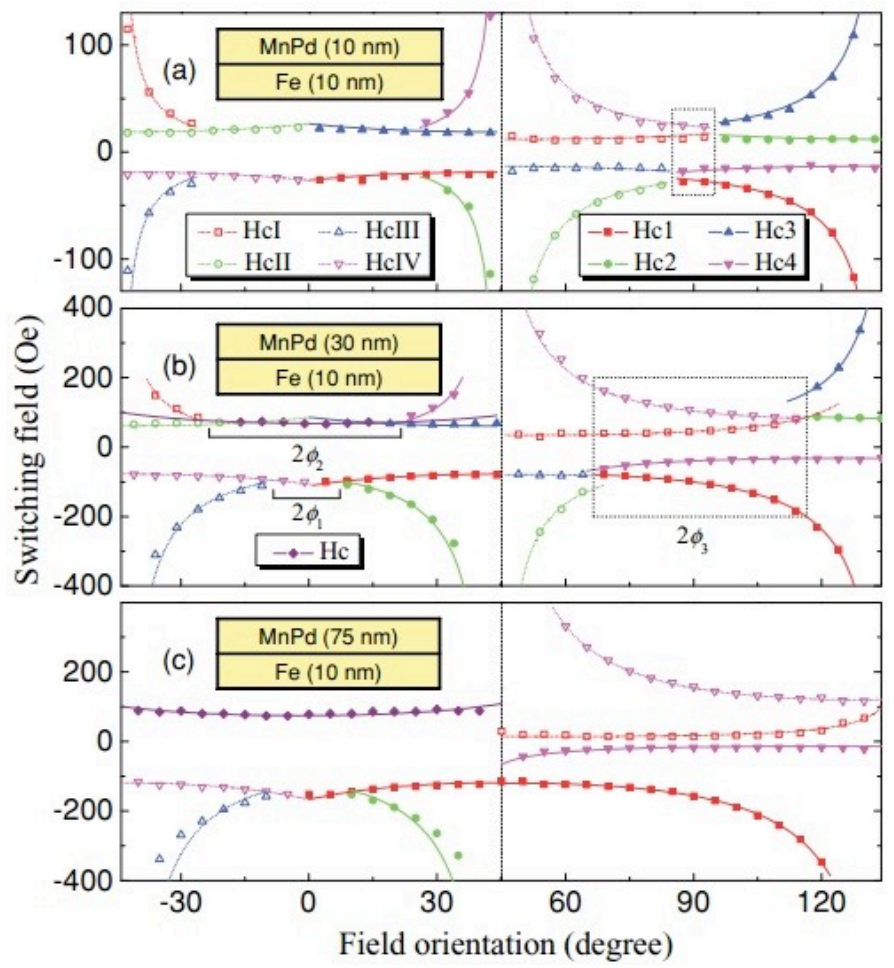

FIG. 17. (Color online) Typical field orientation dependence of the experimentally observed switching fields (symbols) and the corresponding theoretical fitting (curves) to the different switching fields labeled in Fig. 16, for Fe/MnPd bilayers with different MnPd thicknesses. Reprinted from [135], copyright (2011), with permission from APS.

\subsubsection{Merits of the model}

Besides the precise modeling of the switching fields under the DW nucleation mechanism, this model also offers unique insights into EB related properties in a more general sense, which are applicable also for epitaxial samples grown along non-(002) orientations, as well as for some polycrystalline systems. For example, the famous asymmetrical loop, with two-step reversal for one branch and one-step reversal for the other branch [Fig. 18(a)], observed for many EB bilayers can be nicely explained by the fact that the two-consecutive $90^{\circ} \mathrm{DW}$-type reversals are inequivalent, i.e. $H_{\mathrm{c} 1}$ and $H_{\mathrm{c} 2}$, for the descending branch, but for the ascending branch are degenerate with a single value, i.e. $H_{\mathrm{c}}$. In polycrystalline samples, the different switchings convolute with each other due to a broader switching field distribution and such asymmetry is usually unpronounced. In epitaxial samples, the stepped features are easily recognized and can be modeled due to the sharp magnetic transitions occurring via DW type reversal. 
In addition, specific details about the exact type of DW nucleation can sometimes be only distinguished by fitting its angular dependence under the DW nucleation model. For example, Fig. 18(b) summarizes the angular dependent switching fields around the bias direction $\left(-45^{\circ}<\varphi<\right.$ $\left.45^{\circ}\right)$ for thin $(10 \mathrm{~nm})$ and thick $(75 \mathrm{~nm}) \mathrm{MnPd}$ samples, respectively. For the thick samples, the angular dependence exhibits a smooth ' $\cos (\varphi)$ '-type of behavior across the $-45^{\circ}<\varphi<45^{\circ}$ range and is consistent with a $180^{\circ} \mathrm{DW}$ reversal; however, the angular dependence for the thin sample exhibits a ' $\sin (\varphi)+\cos (\varphi)$ '-type of behavior with a first-order transition at $\varphi \sim 0^{\circ}$, which is in agreement with two consecutive $90^{\circ} \mathrm{DW}$ reversals. As a result, despite the fact that both samples show a one-step transition as seen from the hysteresis loops, the underlying reversal mechanisms are completely different as evidenced from their angular dependences. Such effects are normally masked out in polycrystalline EB systems.
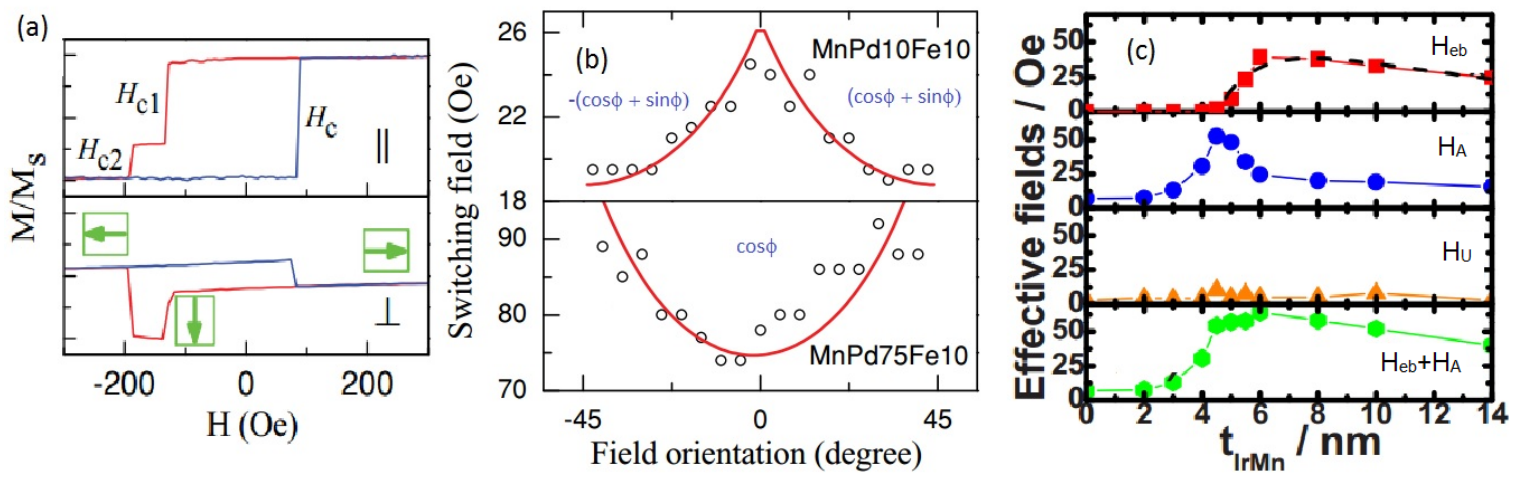

FIG. 18. (Color online) (a) Typical longitudinal $(\|)$ and transverse $(\llcorner)$ MOKE loops measured for the bilayer with $t_{\mathrm{MnPd}}=75 \mathrm{~nm}$ and at $\varphi=20$. An asymmetrically shaped loop is observed. The red and the blue curves correspond to the descending and the ascending branches of the hysteresis loops, respectively. (b) The detailed view of the angular dependence of the switching fields (upper panel) $\mathrm{H}_{\mathrm{cII}}$ and $\mathrm{H}_{\mathrm{c} 3}$ for the $\mathrm{MnPd} / \mathrm{Fe}$ bilayer with $\mathrm{t}_{\mathrm{MnPd}}=10 \mathrm{~nm}$ and (lower panel) $\mathrm{H}_{\mathrm{c}}$ for the sample with $\mathrm{t}_{\mathrm{MnPd}}=75 \mathrm{~nm}$. They can be interpreted by the magnetic reversal mechanism of two successive $90^{\circ} \mathrm{DW}$ nucleations and a $180^{\circ} \mathrm{DW}$ nucleation, respectively. Reprinted from [135], copyright (2011), with permission from APS. (c) Thickness dependence of the anisotropies in epitaxial Fe/IrMn systems. Reprinted from [35], copyright (2011), with permission from AIP.

Another advantage of this model is the direct extraction of the dependence of key anisotropies on external parameters (temperature, thickness, etc.), as well as their unambiguous correlation with the microscopic interface spin behavior owing to their distinct symmetry (cubic, uniaxial, or unidirectional). For example, Fig. 18(c) shows the extracted thickness dependence of the anisotropies in the epitaxial Fe/IrMn bilayers. It is noted that for $\mathrm{t}_{\mathrm{IrMn}}<4 \mathrm{~nm}$, the exchange anisotropy, $H_{\mathrm{eb}}=0$ and the ferromagnetic rotational anisotropy, $H_{\mathrm{A}}$ displays a gradual enhancement with $\mathrm{t}_{\mathrm{IrMn}}$. For $4 \mathrm{~nm}<\mathrm{t}_{\mathrm{IrMn}}<6 \mathrm{~nm}, H_{\mathrm{eb}}$ rapidly increases, however, $H_{\mathrm{A}}$ decreases after reaching a peak at $\mathrm{t}_{\mathrm{IrMn}}=4.5 \mathrm{~nm}$. Interestingly, the sum $H_{\mathrm{eb}}+H_{\mathrm{A}}$, remains at almost the same value for the range $4.5 \mathrm{~nm}<\mathrm{t}_{\mathrm{IrMn}}<6 \mathrm{~nm}$. Such results points directly to the competing effects of the pinned and 
rotatable AF spins at the interface. For $\mathrm{t}_{\mathrm{IrMn}}<4 \mathrm{~nm}$, the AF anisotropy is too weak to establish the bias. The AF spins at the interface only reverse with the F spins due to the strong exchange coupling, and contribute to the enhanced rotatable $\mathrm{F}$ anisotropy, $H_{\mathrm{A}}$, rather than being pinned and contribute to $H_{\mathrm{eb}}$. As $\mathrm{t}_{\mathrm{IrMn}}$ further increases, certain $\mathrm{AF}$ spins start to be pinned to contribute to $H_{\mathrm{eb}}$ while $H_{\mathrm{A}}$ keeps increasing until $t_{\mathrm{IrMn}}=4.5 \mathrm{~nm}$. Since the $\mathrm{EB}$ is an interfacial effect, the total number of pinned and rotatable spins reaches saturation at a certain $t_{\mathrm{IrMn}}$ value, which is $4.5 \mathrm{~nm}$ in our case. Further increasing $t_{\mathrm{IrMn}}$ only results in more AF spins being pinned and less of them being rotatable. In Section 3, the different depth profiles and the competing effects of the pinned and rotatable AF spins at the EB interface have been introduced. Direct observations of such spin magnetic moments normally require non-trivial synchrotron radiations such as XRMR technique [54]. Here it is shown that by this analytical model such spin information can also be extracted indirectly from their representative anisotropies.

To briefly summarize, the "DW nucleation model" begins with surveying all the possible anisotropies in a magnetic system and focusing on the anisotropy symmetries and their respective orientations. It has proved to be extremely successful in modeling epitaxial EB systems due to their pronounced anisotropies and distinct magnetization reversals. Quantitatively, it models the magnitude of reversal fields very well, and is also able to distinguish tiny stepped reversal features (sometimes on the order of a few Oe) due to the multistage DW nucleation processes. This is partially due to the fact that such a model directly unravels the switching fields from balancing different anisotropies, unlike the conventional Meiklejohn-Bean (MB) approach which primarily models the hysteresis loop. In addition, the various magnetic anisotropies, either intrinsic or induced, find their origins in the crystal (magnetocrystalline), shape and/or interface characteristics. The "DW nucleation model" allows practical film properties such as the microstructures and their roles in magnetic reversals to be included in the modeling. For example, the DW nucleation energies are treated as fitting parameters which effectively contain contributions from magnetocrystalline anisotropy, detects, pinning potential, etc. Furthermore, the induced anisotropies are directly linked to interfacial spin magnetic moments, providing access to the pinned and rotatable behaviors of the surface moments and their dependence over temperature, field cooling, training, and annealing effects. Last but not least, the current version of this model only considers a single type of DW nucleation process for a particular reversal, i.e. either $90^{\circ}$ or $180^{\circ}$, and neglects any potential switching distribution that may be involved due to inhomogeneity. As a result, for some EB systems with mixed coherent rotation and DW nucleation mechanisms and those with larger film inhomogeneity, the current "DW nucleation model" may be found 
wanting due to incomplete access to the microscopic details of the samples. A more generalized model may be necessary which also points to a possible direction for future study.

\section{Future Perspectives}

Complementing the advances in the field of exchange bias in which AFs are only passively used for pinning the Fs, in the past decade, AFs have also found many emergent applications in spintronics where they have been playing a much more active role. A particular exciting example is the direct electrical switching of an antiferromagnet, CuMnAs [216], a metal with a highly specific, non-centrosymmetric crystal structure [217]. Such discovery makes it truly appealing and promising to constructing future all-antiferromagnetic memory devices [218]. The unique advantages of antiferromagnets, such as zero net magnetization, nontrivial spin-orbit coupling, and non-collinear magnetism, have made them attractive and promising for robust spin-based electronics devices and higher-frequency applications beyond ferromagnetic resonance [219-225]. Currently, major research endeavors along these lines take advantage of their robust yet tunable antiferromagnetic ordering, appreciable spin-orbit interactions, and other non-relativistic and relativistic spin-transport phenomena. Since most of the novel functionalities discussed below require well-ordered antiferromagnetic spin moments, it is obvious that these future studies would benefit enormously from epitaxial grown antiferromagnetic heterostructures, with well-defined, and possibly optimized structural and magnetic properties. The following sections are meant to briefly walk the reader through some of the interesting recent developments.

\subsection{Anisotropic-magnetoresistance in antiferromagnets}

Magnetoresistance phenomena are the fundamental basis for current and future magnetic storage technologies. These effects have been well known and extensively studied in ferromagnets. Recent experiments have shown that such effect is not unique to ferromagnets but also exists in antiferromagnets. Park et al demonstrated tunneling anisotropic magnetoresistance in a vertical stack in which antiferromagnetic IrMn is placed between $\mathrm{MgO}$ and $\mathrm{NiFe}$ [223]. A large spin-valvelike signal was observed, which is attributed to relative change of the orientation of the IrMn and NiFe moments. This initial experiment, illustrated in Fig. 19, established the fundamental picture of using magnetotransport effects for possible information storage utilizing an antiferromagnet. 
Such observation has been quickly extended to room temperature and in a perpendicular anisotropy system [226].
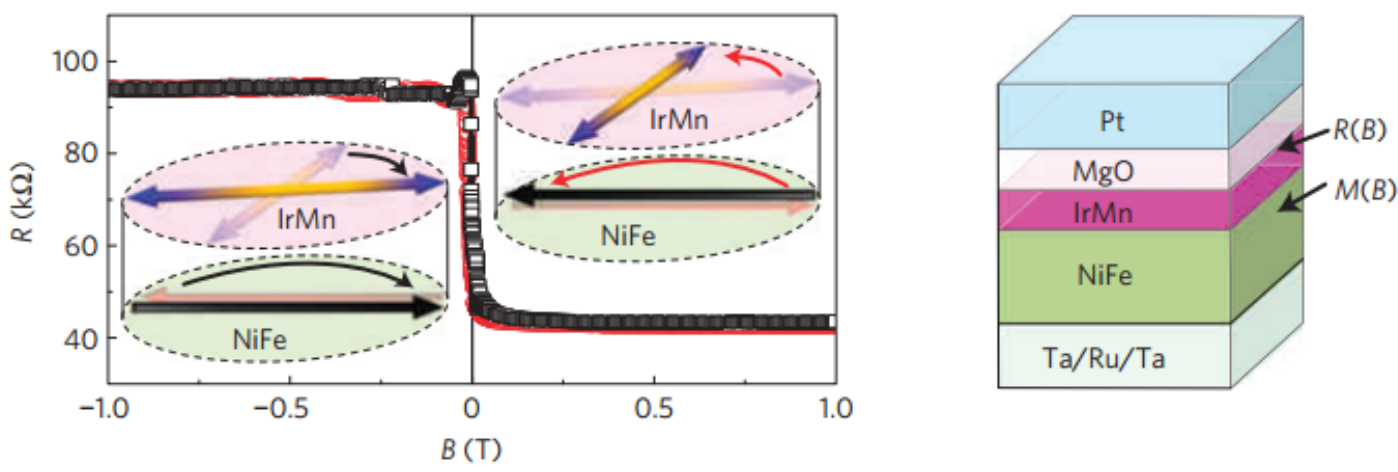

FIG. 19. (Color online) A spin-valve-like signal in the $\mathrm{NiFe} / \mathrm{IrMn}(1.5 \mathrm{~nm}) / \mathrm{MgO} / \mathrm{Pt} \mathrm{AF}$ tunnel device compared with the weak magnetoresistance of an $\mathrm{F} \mathrm{NiFe} / \mathrm{MgO} / \mathrm{Pt}$ tunnel junction. A $130 \%$ magnetoresistance signal recorded in the range of -1 to $+1 \mathrm{~T}$ field on a tunnelling device fabricated in the depicted multilayer structure with the $\mathrm{NiFe} / \mathrm{IrMn}(1.5$ $\mathrm{nm}) / \mathrm{MgO} / \mathrm{Pt}$ tunnel junction. The direction of the in-plane magnetic field corresponds to the direction of the magnetic field applied during the film growth. The insets illustrate the rotation of AF moments in IrMn through the exchangespring effect of the adjacent $\mathrm{NiFe}$ ferromagnet. The external magnetic field is sensed by the NiFe ferromagnet whereas the tunnelling transport is governed by the IrMn antiferromagnet. Reprinted from [223], copyright (2011), with permission from Nature publishing group.

Similar anisotropic magnetotransport behavior was also observed later in semiconducting iridates, $\mathrm{Sr}_{2} \mathrm{IrO}_{4}$, [227,228] and $\mathrm{FeRh}[229,230]$, as depicted in Fig. 20. Iridates belong to a new class of insulator, namely the spin-orbit insulator. By using point-contact geometry, an AMR ratio as large as $14 \%$ has been measured. In addition, the complex and tunable (by external fields) angular dependence of the AMR signal suggests its stronger correlation with the materials electronic states. FeRh has a temperature driven F-AF transition at $\sim 400 \mathrm{~K}$, at which an applied magnetic field $\left(H_{\mathrm{FC}}\right)$ can align the magnetization along either [010] or [100] crystal direction; after cooling with $H_{\mathrm{FC}}$ to temperatures below the F-AF transition temperature, the AF spin axis will be locked at [100] or [010], respectively, giving rise to binary resistance states that can be used for information storage and processing (Fig. 20). 

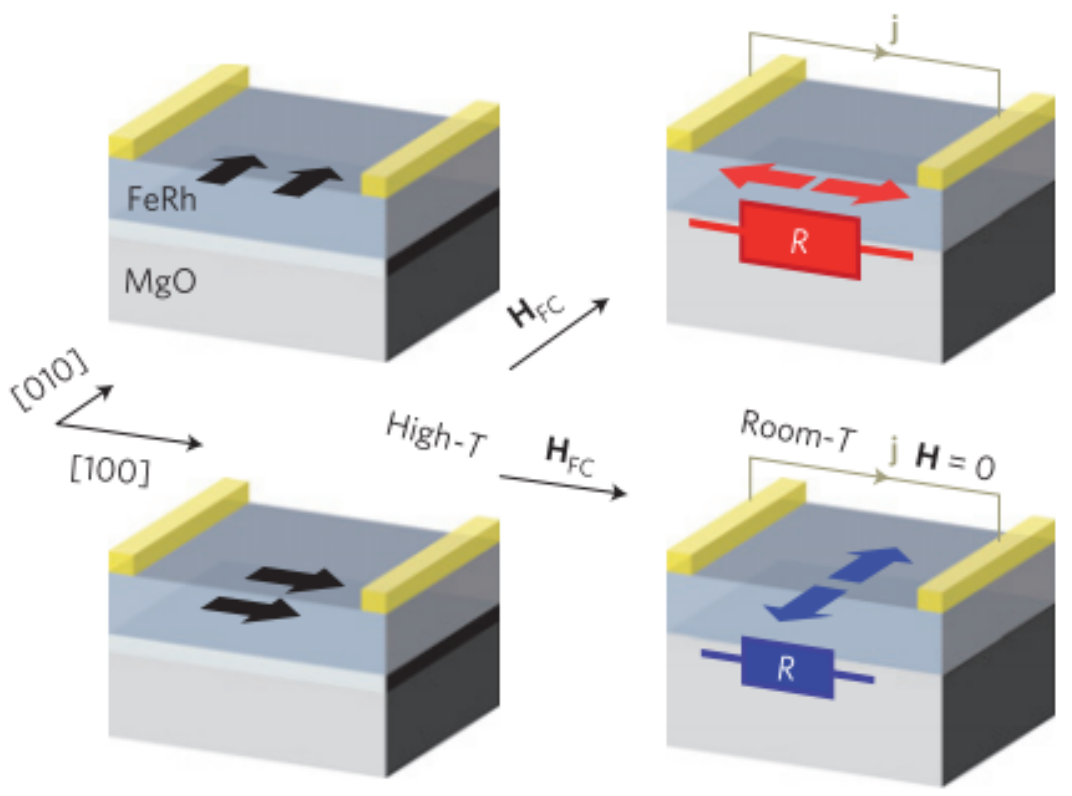

FIG. 20. (Color online) Schematic illustration of the $\mathrm{AF} \mathrm{FeRh/MgO} \mathrm{structure} \mathrm{and} \mathrm{the} \mathrm{set-up} \mathrm{for} \mathrm{the} \mathrm{memory} \mathrm{writing}$ reading. For writing, the sample is cooled in a field $H_{\mathrm{FC}}$ from a temperature above the $\mathrm{AF}-$ ferromagnetic transition in FeRh (a maximum field of $9 \mathrm{~T}$ and temperature of $400 \mathrm{~K}$ was used) to below the transition temperature $(200 \mathrm{~K})$. Black arrows denote the orientation of the magnetic moments in the ferromagnetic phase whereas either red or blue arrows denote two distinct configurations of the magnetic moments in the AF phase. The resulting AF spin axis in the lowtemperature memory state depends on the direction of $H_{\mathrm{FC}}$, which is either along the [100] or [010] crystal axis. For reading, electrical current, $\mathrm{j}$, is driven between electrical contacts (yellow bars) along the [100] direction and the resistance is detected. Reprinted from [229], copyright (2014), with permission from Nature publishing group.

\subsection{Anomalous- and Spin-Hall effects of antiferromagnets}

Generation of spin-polarized current is the key to virtually all spintronics concepts, which is usually achieved by passing a charge current through a ferromagnetic polarizer. More recently it has been realized that spin-orbit interaction can provide an alternative but efficient pathway for the same purpose, even when using non-magnetic conductors. Among the key phenomena arising from spin-orbit interactions is the spin Hall effects which was theoretically predicted in 1971 [231]. Such effects convert an initially unpolarized charge current into a transverse spin current, resulting in spin accumulation at the boundaries of the conductor. A materials-specific spin Hall angle, given by the ratio of charge-to-spin current densities [232,233], is a good measure of the conversion efficiencies. For example, Liu et al demonstrated that the spin Hall angle in $\mathrm{Ta}(\sim 12 \%)$ is sufficiently large to switch the magnetization in a tunnel junction [234], indicating such effects can have significant application as a source for spin currents. 

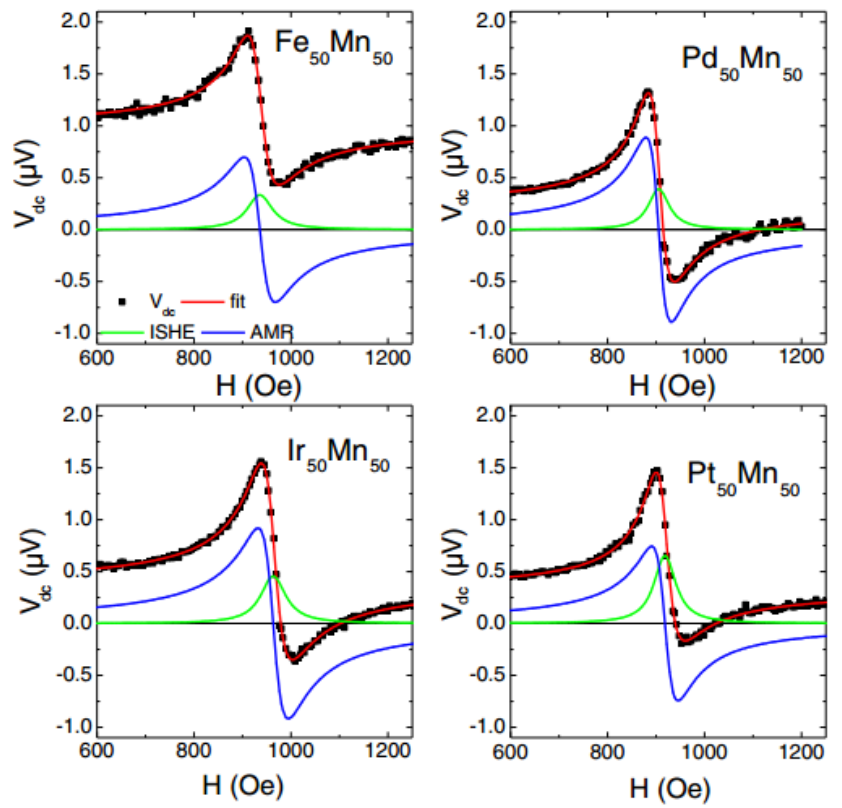

FIG. 21. (Color online) Room temperature spin pumping and inverse spin Hall effect experiments conducted at 9 $\mathrm{GHz}$ for $\mathrm{Py}(15) / \mathrm{Cu}(4) / \mathrm{AF}(5)$ structures where $\mathrm{AF}=\mathrm{FeMn}, \mathrm{PdMn}, \mathrm{IrMn}$, and PtMn. Reprinted from [240], copyright (2014), with permission from APS.

Large anomalous Hall effects and spin Hall effects have also been proposed for antiferromagnetic $\mathrm{FeMn}, \mathrm{IrMn}_{3}, \mathrm{Cr}$, and $\mathrm{Mn}_{2} \mathrm{Au}$, owing to the large atomic spin-orbit coupling from the heavy elements as well as the Berry phase from their non-collinear spin textures [235-239]. For example, sizable spin-Hall effects have been revealed in CuAu-I-type metallic antiferromagnets, FeMn, PdMn, IrMn, and PtMn, as shown in Fig. 21, by using spin pumping [240] and spin Seebeck measurements [224]. The estimated spin-Hall angles of the four materials follow the relationship PtMn $>$ IrMn $>$ PdMn $>$ FeMn, corroborating the important role of the spin-orbit coupling of the heavy metals [241] for the properties of the Mn-based alloys through orbital hybridization. In addition, first-principles calculations also indicate strong influence from the staggered magnetization of $\mathrm{Mn}$ on the intrinsic spin-Hall effect of these alloys, suggesting the possibility of even anisotropic spin-orbit effects in these AF materials. It is worth emphasizing that to experimentally realize such anisotropic effects it requires epitaxial growth of these AF alloys [242].

\subsection{Spin orbit torque and spin Hall magnetoresistance}

Recently, heavy-metal/magnetic-insulator structures have received increasing attention due to a newly proposed magnetoresistance at such interfaces, i.e. spin Hall magnetoresistance (SMR). 
Such magnetoresistance takes advantage of the field-invariant spin current generation in the heavy metal due to spin-orbit effects (e.g. spin Hall effects), as well as the different efficiencies of spin current absorption due to field-variant magnetization direction of the magnetic insulator. As a result, although the conduction electrons cannot enter the magnetic insulator, the bilayer resistance can still reflect its magnetization direction due to such a non-equilibrium proximity effect at the interface. First discovered in YIG/Pt [243], such phenomena have been quickly extended to other systems not limited to insulators [244,245]. Since such magnetoresistance, in principle, only utilize the interfacial magnetic ordering and weakly depends on bulk ferromagnetic order, other magnetically ordered interfaces, including antiferromagnetic ones, should also exhibit similar effects. The spin Hall magnetoresistance at an antiferromagnetic interface has been recently observed in $\mathrm{SrMnO}_{3} / \mathrm{Pt}$ systems [246], as shown in Fig. 22. The magnetoresistance ratio is around $0.01 \%$, which is comparable to Pt/YIG [243] and even larger than that for Pd/YIG interfaces $(0.001 \%)$ [247]. Such results also indicate that the key for observing SMR lies in the heavy-metal and the magnetic interfaces, but is only less influenced by the bulk magnetic insulator. In this sense, it is needless to say that high quality interfaces from epitaxially grown samples are indispensable for such observations, since the spin transfer torque needs to be coherently absorbed by the interfacial spins across the whole heavy-metal structure. Finally, although the work presented in Fig. 22 still uses an external magnetic field for the manipulation of SMO moments, such process can also be achieved via other coupling mechanism such as electrical gating effect [248]. The spin Hall magnetoresistance at the antiferromagnetic interface could enable spin-based signal generation and detection without any ferromagnetic components. Therefore, they hold promise for high density memory integration free from perturbations caused by stray field and/or external magnetic field. 

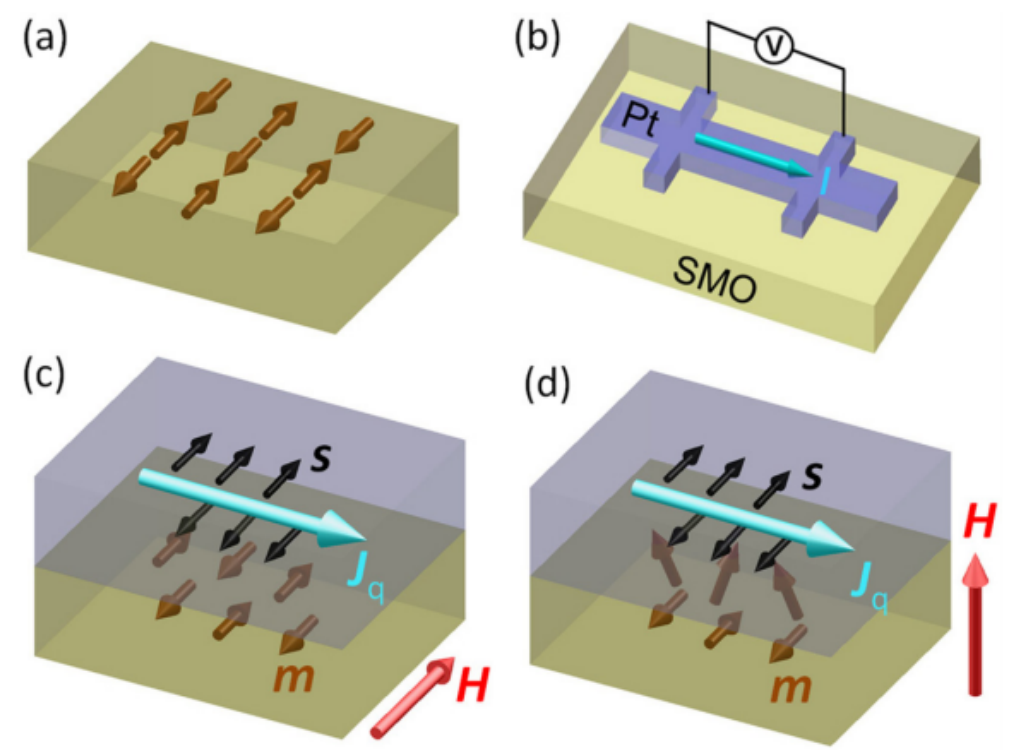

FIG. 22. (Color online) (a) The AFM moment arrangement in the (001) plane of SMO. (b) A schematic of the sample layout. (c) Low resistance state at the zero field or with in-plane $H$. (d) High-resistance state with out-ofplane $H$. The SMR is determined by the relative arrangement between the interfacial electron spins(s) in Pt and the AFM moments (m) in SMO. Reprinted from [246], copyright (2014), with permission from APS.

\subsection{Spin current transmission through antiferromagnets}

The strong spin-orbit interaction of metallic antiferromagnets indicates that they are suitable candidate for spin current detection. However, it also implies that the spin current would quickly dephase in these materials. An earlier report [240] has suggested that the spin diffusion length of metallic antiferromagnets are rather short, $\sim 1-2 \mathrm{~nm}$, which makes them unfavorable for transmitting spin information over long distance. This scenario can be improved by coupling the spin current to antiferromagnetic magnons. Takei et al [249] theoretically evaluated the possibility of transmitting spin currents across antiferromagnets, where a coherent antiferromagnetic ordering is considered for magnon-mediated spin transport, and they arrived at an exponential decay of the spin voltage over the spin diffusion length and/or the AF healing length (determined by the AF anisotropies). Wang et al [250] and Hahn et al [251] experimentally investigated spin current transmission across insulating antiferromagnetic $\mathrm{NiO}$ by using spin pumping as a spin current source. They clearly demonstrated highly efficient spin transport across the AFs. Another followup work by Wang et al [252] also investigated a series of insulating AFs, which verified the exponential decay of spin current, yielding decay lengths ranging from several $\AA$ to several nm; see Fig. 23. In particular, the decay length for $\mathrm{NiO}$ is $\sim 10 \mathrm{~nm}$, standing out amount other AFs investigated. On the other hand, Moriyama et al [253] adopted spin-torque ferromagnetic 
resonance technique, which is the reciprocal experiment to spin pumping, and independently confirmed spin-orbit torques through an epitaxial grown NiO. Their results also suggest that certain structural/crystalline symmetries have to be avoided or met to realize efficient transmission, highlighting the importance of epitaxial structures for optimal device performance. In the case of $\mathrm{NiO},(001)$ is more favorable than the (111) orientation due to the strong magnetic easy-plane along (111) that hinders the coherent Néel order dynamics. Similar result was also achieved later by Lin et al using spin Seebeck effect experiments [254]. The detailed mechanisms for spin current in AFs remain to be understood [255-257], but it is qualitatively believed that the magnetic excitations in the insulators are expected to play a major role, for example, in the form of precessional spin wave modes that are strongly dependent on either the static AF ordering or the fluctuating correlated moments.
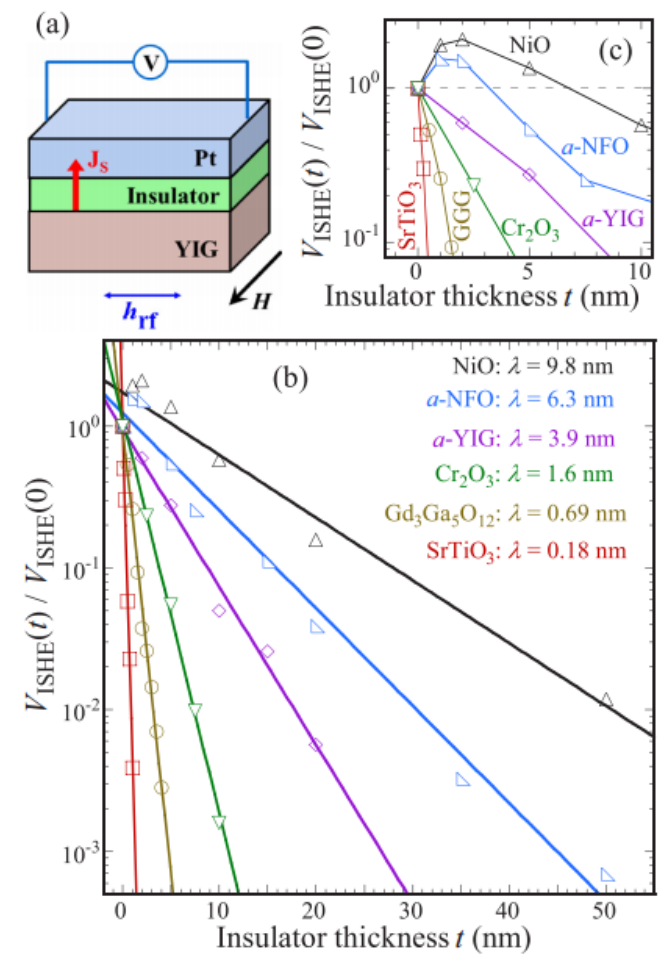

FIG. 23. (Color online) (a) Schematic of the spin pumping measurement on various Pt/insulator/YIG structures. (b) Semilog plots of the inverse spin-Hall voltage, $\mathrm{V}_{\mathrm{ISHE}}$, as a function of the insulator thickness for the six series normalized to the values for the corresponding Pt/YIG bilayers, where the straight lines are exponential fits to each series, from which the spin decay lengths $\lambda$ are determined. (c) Details of behavior shown in (b) for insulators below $10 \mathrm{~nm}$. Reprinted from [252], copyright (2015), with permission from APS.

\subsection{Spin current generation through antiferromagnets}



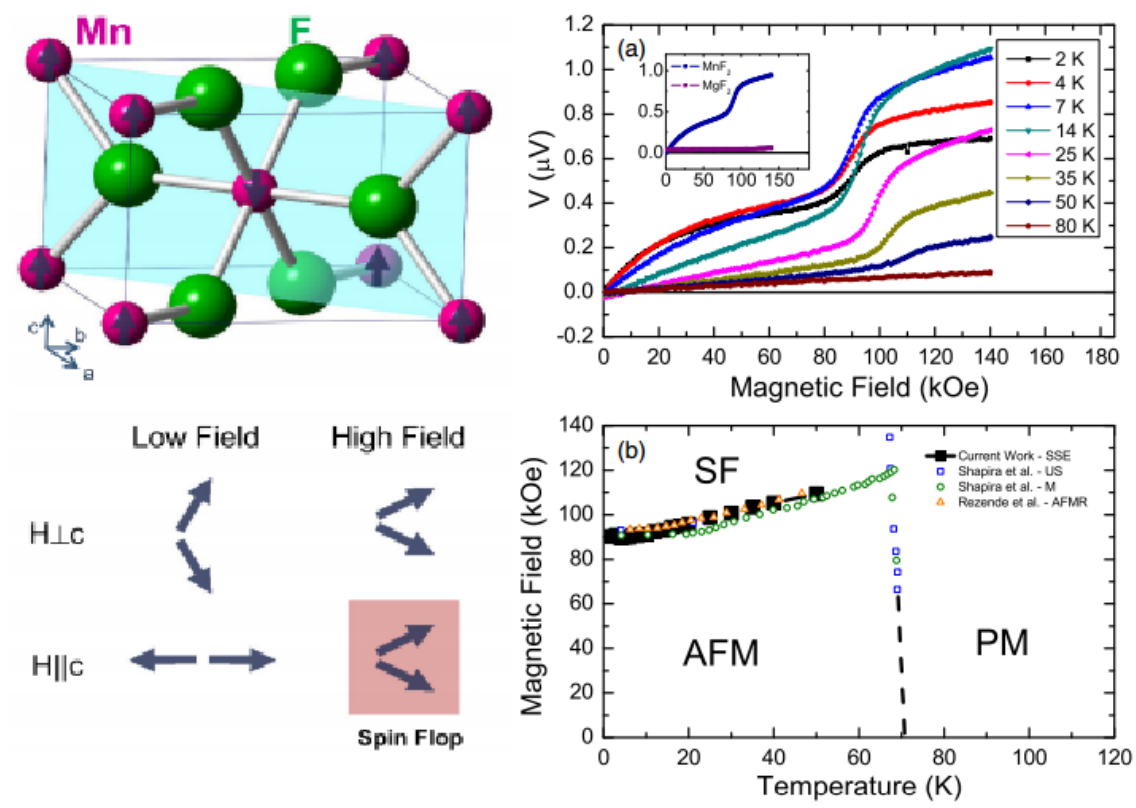

FIG. 24. (Color online) (left) The crystal structure of $\mathrm{MnF}_{2}$ is presented with $\mathrm{AF}$ spin structure overlaid on $\mathrm{Mn}^{2+}$ ions. The (110) thin film crystal orientation plane is highlighted in blue. The spin-flop transition in $\mathrm{MnF}_{2}$ is presented. (right) Spin Seebeck voltage response curves from $\mathrm{MnF}_{2}$ are shown with magnetic field applied parallel to the $c$-axis in (a). A control experiment is performed with a bare $\mathrm{MgF}_{2}$ substrate with $\mathrm{Cu}(2 \mathrm{~nm}) / \mathrm{Pt}(4 \mathrm{~nm})$ under the same conditions at $5 \mathrm{~K}$ showing no measurable effect (inset). By mapping the spin-flop transition from (a), a phase diagram for $\mathrm{MnF}_{2}$ is reproduced in (b). These data are compared to data from earlier studies using other techniques. Reprinted from [259], copyright (2016), with permission from APS.

Pumping spin currents out of antiferromagnets would be one of the ultimate goals for AF spintronics, which can be potentially realized by spin Seebeck effect [258,259] and/or spin pumping effects [260]. Spin Seebeck effect is the generation of a spin current by a thermal gradient, which is the key phenomenon in the field of spin-caloritronics [261]. Experimental realization of the spin Seebeck effect in an AF has been recently demonstrated in $\mathrm{MnF}_{2}$ [259] and $\mathrm{Cr}_{2} \mathrm{O}_{3}$ [262]. In both cases, the simultaneously applied large in-plane magnetic field and the longitudinal thermal gradient caused appreciable spin-Seebeck voltage along the Pt detection layer at the spin-flop condition of $\mathrm{MnF}_{2}$ and $\mathrm{Cr}_{2} \mathrm{O}_{3}$; see Fig. 24. Further theoretical work suggests a significant contribution from bulk antiferromagnetic magnon spin current, created by the temperature gradient across the thickness of the $\mathrm{MnF}_{2} / \mathrm{Pt}$ or $\mathrm{Cr}_{2} \mathrm{O}_{3} / \mathrm{Pt}$. In particular, the spin currents carried by the two magnon modes (due to the two sublattices) have opposite directions, which speaks for the necessity of large external magnetic field for the production of a net spin current [263].

Experimental realization for $\mathrm{AF}$ spin pumping would require resonating $\mathrm{AF}$ spin magnetic moments, which further requires large magnetic fields, on the order of several to tens of Tesla, and 
ultrahigh exciting frequency, on the order of THz [264]. With the rapid developments in modern optics and advanced electronics, realization of antiferromagnetic resonance has become promising in the past several years. Once readily available, antiferromagnets can, in principle, replace ferromagnets, and when they do an all-AF-based spin-electronics will become possible, in which AFs actively perform all functions including spin current sourcing, transmitting, and detecting.

\section{SUMMARY}

The purpose of this review is to walk the reader through recent works on exchange bias, post $2000 \mathrm{AD}$, and try to make the bridge between past achievements and the promise of ongoing developments in the field of antiferromagnetic spintronics. It is unnecessary and impossible to cover all aspects related to exchange bias and the review is not meant to be exhaustive. Therefore, we build on previous reviews and use them as a spring board to focus on epitaxial EB systems, including their growth, characterizations, and properties. We discuss, in depth, two aspects related to exchange bias that benefit from the study of epitaxial samples, i.e. the unambiguous interfacial spin information and the distinct yet pronounced magnetic anisotropies. Both aspects are difficult to address in polycrystalline samples.

In the first part of the review, we discuss the interface structures and their manifestation in terms of transport, x-ray, imaging measurements, etc. While the rotatable and pinned spins in the antiferromagnets, as directly revealed by synchrotron experiments, are the primary source for measured magnetic behaviors, more complex interface spin texture can also arise due to competing anisotropies. The long range ordering also makes such samples sensitive to local defects and microstructures. In the second part, we move our focus to magnetic anisotropies in these systems. We begin with a survey of different symmetries of the magnetic anisotropies and further show their competing effects in terms of magnetic reversal. Finally, we introduce a quantitative model based on domain-wall processes and bridge the microscopic spin behavior with the various induced anisotropies.

Last but not least, while developments of epitaxial exchange bias systems offer new platforms for the study of novel exchange bias phenomena, such ferromagnetic/antiferromagnetic heterostructures are also indispensable in almost all the emerging fields that are relevant to antiferromagnetic spintronics. In the last part, we extend our discussion to a broader perspective and show some emerging discoveries using similar F/AF structures, such as novel 
magnetoresistance and spin current related phenomena. These new directions highlight the important role of coherent spin textures and long range antiferromagnetic ordering in the sense of new functionalities such as spin current generation and transportation. In the future, these new functionalities may be further convoluted with the study of exchange bias and other interface properties, and by various measurement approaches such as those via optical and electrical means. As a result, the field of exchange bias, broadly defined to include both magnetic phenomena and spin transport, is expected to see continuous developments and advances driven by the intriguing properties of antiferromagnets.

\section{ACKNOWLEDGEMENTS}

W.Z. is very grateful for the enormous help, support and encouragement received from his collaborators throughout these years: Dr. Qingfeng Zhan (NIMTE), Dr. Mark Bowden (PNNL), Dr. Sebastian Bruck (West Australia), Dr. Thomas Eimuller (Kempten), Dr. Yu Fu (Duisburg), Prof. Mingzhong Wu (CSU), Dr. Matt Ferguson (UW), Dr. Yufeng Hou (Western Digital), Dr. Zheng Li (Apple), Dr. Axel Hoffmann (ANL), Dr. Suzanne te Velthuis (ANL), and Dr. Yaohua Liu (ORNL). We also thank NSF for financial support under grant No. 1063489. Work at Argonne, including finalization of the manuscript, is supported by the U.S. Department of Energy, Office of Science, Materials Science and Engineering Division.

\section{REFERENCES}

[1] W. P. Meiklejohn and C. P. Bean, Phys. Rev. 102, 1413 (1956).

[2] Review. J. Nogues and I. K. Schuller, J. Magn. Magn. Mater. 192, 203 (1999).

[3] Review. A. E. Berkowitz and K. Takano, J. Magn. Magn. Mater. 200, 552 (1999).

[4] Review. M. Kiwi, J. Magn. Magn. Mater. 234, 584 (2001).

[5] Review. R. L. Stamps, J. Phys. D: Appl. Phys. 33, R247(2000).

[6] Review. J. Nogues et al., Phys. Rep. 422, 65 (2005).

[7] Review. K. O’Grady et al., J. Magn. Magn. Mater. 322, 883 (2010).

[8] A. K. Suszka et al., Phys. Rev. Lett. 109, 177205 (2012).

[9] E. Mlynczak et al., Phys. Rev. B 88, 085442 (2013).

[10] Review. Krishnan et al., J. Mater. Sci. 41, 793 (2006).

[11] Review. G. Srajer et al., J. Magn. Magn. Mater. 307, 1 (2006).

[12] Review. M.R. Fitzsimmons et al, J. Magn. Magn. Mater. 271, 103 (2004).

[13] Mathieu et al, J. Appl. Phys. 83, 2863 (1998).

[14] A. Brambilla et al., Thin Solid Films, 516, 7519 (2008).

[15] F. Radu et al., Phys. Rev. B 79, 184425 (2009).

[16] R. M. Wolf et al., Mater. Res. Soc. Symp. Proc. 341, 23 (1994).

[17] Y. Ijiri et al., Phys. Rev. Lett. 99, 147201 (2007).

[18] W. Zhu et al., Phys. Rev. Lett. 86, 5389 (2001). 
[19] J. Wu et al., Phys. Rev. B 80, 012409 (2009).

[20] M. Finazzi et al., Phys. Rev. B 69, 014410 (2004).

[21] R. Jungblut et al, J. Appl. Phys. 75, 6659 (1994).

[22] C. Liu et al., J. Appl. Phys. 87, 6644 (2000).

[23] A. Tan et al, Phys. Rev. B 88, 104404 (2013).

[24] T. Lin et al., Appl. Phys. Lett. 65, 1183 (1994).

[25] S. Mao et al., Appl. Phys. Lett. 69, 3593 (1996).

[26] V. Cantelli et al., J. Appl. Phys. 99, 08C102 (2006).

[27] K. A. Seu et al, J. Appl. Phys. 93, 6611 (2003).

[28] G. Vinai et al, Appl. Phys. Lett. 104, 162401 (2014).

[29] Y. F. Liu et al, J. Phys. D: Appl. Phys. 42, 115002 (2009).

[30] J. Sort et al, Phys. Rev. B 71, 054411 (2005).

[31] A. Kohn et al, Sci. Rep. 3, 2412 (2013).

[32] S. G. Wang et al., J. Phys. D: Appl. Phys. 42, 225001 (2009).

[33] N. N. Phouc et al., IEEE. Trans. Magn. 44, 2828 (2008).

[34] H. H. Huang et al., J. Appl. Phys. 105, $07 D 724$ (2009).

[35] W. Zhang et al., Appl. Phys. Lett. 98, 092503 (2011).

[36] J. Nogues et al., Phys. Rev. B 59, 6984 (1999).

[37] N. Cheng et al., J. Appl. Phys. 89, 6597 (2001).

[38] P. Blomqvist et al., J. Appl. Phys. 96, 6523 (2004).

[39] J. Choi et al., Phys. Rev. B 76, 054407 (2007).

[40] S. Roy et al., Phys. Rev. Lett. 95, 047201 (2005).

[41] J. Wu et al., Phys. Rev. Lett. 104, 217204 (2010).

[42] Y. Meng et al., Appl. Phys. Lett. 98, 212508 (2011).

[43] J. S. Park et al., Appl. Phys. Lett. 97, 042505 (2010).

[44] M. Pilard et al., Phys. Rev. B 76, 214436 (2007).

[45] E. Blackburn et al., Phys. Rev. B 78, 180408(R) (2008).

[46] S. K. Mishra et al., Phys. Rev. B 81, 212404 (2010).

[47] X. Marti et al., Phys. Rev. Lett. 108, 017201 (2012).

[48] P. Kappenberger et al., Phys. Rev. Lett. 91, 267202 (2003).

[49] I. Schmid et al., Phys. Rev. Lett. 105, 197201 (2010).

[50] N. R. Joshi et al., Appl. Phys. Lett. 98, 082502 (2011).

[51] S. Widuch et al., J. Phys. D: Appl. Phys. 44, 415003 (2011).

[52] L. Wee et al., Phys. Rev. B 69, 134426 (2004).

[53] R. L. Rodrigues-Suarez et al., Phys. Rev. B 83, 224418 (2011).

[54] S. Bruck et al., Phys. Rev. Lett. 101, 126402 (2008).

[55] S. Bruck et al., Phys. Rev. B 81, 134414 (2010).

[56] N. C. Koon, Phys. Rev. Lett. 78, 4865 (1997).

[57] F. Nolting et al., Nature 405, 767 (2000).

[58] H. Ohldag et al., Phys. Rev. Lett. 86, 2878 (2001).

[59] H. Ohldag et al., Phys. Rev. Lett. 87, 247201 (2001).

[60] M. Finazzi et al, Phys. Rev. B 70, 235420 (2004).

[61] P. Blomqvist et al., Phys. Rev. Lett. 94, 107203 (2005).

[62] W. J. Antel et al., Phys. Rev. Lett. 83, 1439 (1999).

[63] M. R. Fitzsimmons et al., Phys. Rev. B 75, 214412 (2007).

[64] G. Van der Laan et al., Phys. Rev. B 83, 064409 (2011).

[65] W. Kim et al., Phys. Rev. B 81, 174416 (2010).

[66] J. Wu et al., Phys. Rev. B 79, 212411 (2009).

[67] W. Zhang et al., Phys. Rev. B 86, 054415 (2012).

[68] P. J. Jensen et al., Phys. Rev. B 66, 220407(R) (2002).

[69] B. Y. Wang et al., Phys. Rev. B 83, 104417 (2011).

[70] W. Kuch et al., Phys. Rev. B 65, 140408(R) (2002).

[71] F. Offi et al., Phys. Rev. B 67, 094419 (2003). 
[72] J. Li et al., Phys. Rev. B 83, 094436 (2011).

[73] J. Li et al., Phys. Rev. B 84, 012406 (2011).

[74] M. Gruyters et al., Phys. Rev. Lett. 100, 077205 (2008).

[75] Y. Meng et al., Phys. Rev. B 85, 014425 (2012).

[76] P. S. Normile et al., Phys. Rev. B 76, 104430 (2007).

[77] M. Ali et al., Phys. Rev. B 77, 134401 (2008).

[78] B. Y. Wang et al., Phys. Rev. B 85, 094412 (2012).

[79] G. Chen et al., J. Appl. Phys. 108, 073905 (2010).

[80] W. N. Cao et al., Appl. Phys. Lett. 98, 262506 (2011).

[81] J. Nogues et al., Phys. Rev. Lett. 76, 4624 (1996).

[82] J. Nogues et al., Phys. Rev. B 61, 1315 (1999).

[83] M. Cheon et al., Appl. Phys. Lett. 90, 012511 (2007).

[84] M. Blamire et al., Nat. Mater. 5, 87, (2006).

[85] C. Gatel et al., Eur. Phys. J. B 45, 157 (2005).

[86] I. P. Krug et al., Phys. Rev. B 78, 064427 (2008).

[87] T. Mewes et al., Appl. Phys. Lett. 84, 3840 (2004).

[88] A. K. Nayak, et al, Nature Mater. 14, 679 (2015).

[89] T. C. Schulthess et al., Phys. Rev. Lett. 81, 4516 (1998).

[90] R. Yanes et al., Phys. Rev. Lett. 111, 217202 (2013).

[91] T. J. Moran et al., Appl. Phys. Lett. 72, (1998).

[92] Q. Zhan et al., Appl. Phys. Lett. 96, 112506 (2010).

[93] J. Li et al., Phys. Rev. Lett. 113, 147207 (2014).

[94] W. Zhang et al., Phys. Rev. B 88, 024428 (2013).

[95] Y. Takamura et al., Phys. Rev. Lett. 111, 107201 (2013).

[96] Y. F. Tian et al., Sci. Rep. 3, 1094 (2013).

[97] A. P. Malozemoff, Phys. Rev. B 35, 3679 (1987).

[98] M. R. Ghadimi et al., Appl. Phys. Lett. 87, 261903 (2005).

[99] I. Schmid et al., Europhys. Lett., 81, 17001 (2008).

[100] M. R. Fitzsimmons et al., Phys. Rev. B 65, 134436 (2002).

[101] F. I. F. Nascimento et al., Phys. Rev. B 80, 144407 (2009).

[102] E. Folven et al., Nano Lett. 12, 2386 (2012).

[103] S. M .Wu et al., Nat. Mater. 9, 756 (2010).

[104] S. M. Wu et al., Phys. Rev. Lett. 110, 067202 (2013).

[105] H. Shi et al., Phys. Rev. B 66, 094426 (2002).

[106] M. T. Hutchings et al., J. Phys. C 3, 307 (1970).

[107] J. Olamit et al., Phys. Rev. B 73, 024413 (2006).

[108] J. Nogues et al., Phys. Rev. B 61, R6455 (2000).

[109] S. Maat et al., Phys. Rev. Lett. 87, 087202 (2001).

[110] W. A. A. Macedo et al., Phys. Rev. B 70, 224414 (2004).

[111] W. A. A. Macedo et al., Phys. Rev. B 78, 224401 (2008).

[112] C. Leighton et al., Phys. Rev. Lett. 86, 4394 (2001).

[113] M. J. Pechan et al., Phys. Rev. B 65, 064410 (2002).

[114] J. Olamit et al., Appl. Phys. Lett. 90, 032510 (2007).

[115] S. Widuch et al, Phys. Rev. B 77, 184433 (2008).

[116] M. R. Fitzsimmons et al., Phys. Rev. Lett. 84, 3986 (2000).

[117] S. Brems et al., Phys. Rev. Lett. 99, 067201 (2007).

[118] F. Radu et al., Phys. Rev. B 67, 134409 (2003).

[119] Z. P. Li et al., Phys. Rev. Lett. 96, 217205 (2006).

[120] I. N. Krivorotov et al., Phys. Rev. B 68, 054430 (2003).

[121] A. Scholl et al., Appl. Phys. Lett. 85, 4085 (2004).

[122] M. R. Fitzsimmons et al., Phys. Rev. B 77, 224406 (2008).

[123] I. V. Roshchin et al., Europhys. Lett. 71, 297 (2005). 
[124] J. Olamit et al., Phys. Rev. B 72, 012408 (2005).

[125] O. Petracic et al., Appl. Phys. Lett. 87, 222509 (2005).

[126] M. Kovylina et al., Appl. Phys. Lett. 98, 152507 (2011).

[127] R. Morales et al., Appl. Phys. Lett. 95, 092503 (2009).

[128] M. R. Fitzsimmons et al., Phys. Rev. B 64, 104415 (2001).

[129] M. S. Lund et al., Phys. Rev. B 76, 104433 (2007).

[130] H. Shi et al., Phys. Rev. B 72, 224417 (2005).

[131] P. Miltenyi et al., Phys. Rev. Lett. 84, 4224 (2000).

[132] J. Hong et al., Phys. Rev. Lett. 96, 117204 (2006).

[133] M. Fecioru-Morariu et al., Phys. Rev. Lett. 99, 097206 (2007).

[134] S. K. Mishra et al., Phys. Rev. Lett. 102, 177208 (2009).

[135] Q. Zhan et al., Phys. Rev. B 83, 094404 (2011).

[136] Z.-P. Li et al., Appl. Phys. Lett. 94, 142503 (2009).

[137] N. B. Weber et al., Phys. Rev. Lett. 91, 237205 (2003).

[138] K. Y. Kim et al., Phys. Rev. B 84, 144410 (2011).

[139] R. Morales et al, Phys. Rev. Lett. 102, 097201 (2009).

[140] D. N. H. Nam et al, Appl. Phys. Lett. 93, 152504 (2008).

[141] Y. Xu et al., Phys. Rev. B 84, 054453 (2011).

[142] M. Kovylina et al., Nanotechnology 21, 175301 (2010).

[143] J. Eisenmenger et al, Phys. Rev. Lett. 94, 057203 (2005).

[144] V. Baltz et al., Phys. Rev. Lett. 94, 117201 (2005).

[145] K. Liu et al., Phys. Rev. B 63, 060403(R) (2001).

[146] G. Malinowski et al., Phys. Rev. B 75, 012413 (2007).

[147] S. Laureti et al., Phys. Rev. Lett. 108, 077205 (2012).

[148] E. Girgis et al., Phys. Rev. Lett. 91, 187202 (2003).

[149] Y. Shen et al., J. Appl. Phys. 91, 8001 (2002).

[150] M. Fraune et al., Appl. Phys. Lett. 77, 3815 (2000).

[151] V. Baltz et al., Appl. Phys. Lett. 84, 4923 (2004).

[152] V. Baltz et al., Appl. Phys. Lett. 96, 262505 (2010).

[153] M. S. Lund et al., Phys. Rev. B 66, 054422 (2002).

[154] M. Ali et al., Phys. Rev. B 68, 214420 (2003).

[155] P. J. van der Zaag et al., J. Appl. Phys.79, 5103 (1996).

[156] Ch. Binek et al., J. Magn. Magn. Mater. 234, 353 (2001).

[157] F. Radu et al., J. Phys.: Condens. Matter 18, L29 (2006).

[158] M. D. Stiles et al., Phys. Rev. B 60, 12950 (2001).

[159] B. Craig et al., J. Appl. Phys. 103, 07C102 (2008).

[160] C. Hou et al., Phys. Rev. B 63, 024411 (2000).

[161] J.-G. Hu et al., Eur. Phys. J. B 40, 265 (2004).

[162] M. Gruyters et al., Phys. Rev. B 63, 052401 (2000).

[163] N. N. Phuoc et al., IEEE Trans. Magn. 43, 897 (2007).

[164] E. Jimenez et al., Appl. Phys. Lett. 95, 122508 (2009).

[165] E. Jimenez et al., Phys. Rev. B 80, 014415 (2009).

[166] P. Miltenyi et al., Appl. Phys. Lett. 75, 2304 (1999).

[167] J. V. Kim et al., Phys. Rev. B 71, 094405 (2005).

[168] J. V. Kim et al., Appl. Phys. Lett. 79, 2785 (2001).

[169] J. V. Kim et al., Phys. Rev. B 61, 8888 (2000).

[170] X. P. Qiu et al., Phys. Rev. Lett. 101, 147207 (2008).

[171] T. R. Gao et al., Phys. Rev. Lett. 99, 057201 (2007).

[172] S. Brems et al., Phys. Rev. Lett. 95, 157202 (2005).

[173] A. G. Biternas et al., Phys. Rev. B 80, 134419 (2009).

[174] H. Magnan et al., Phys. Rev. Lett. 105, 097204 (2010).

[175] M. Fecioru-Morariu et al., Phys. Rev. B 77, 054441 (2008).

[176] D. Spenato et al., Appl. Phys. Lett. 91, 062515 (2007). 
[177] A. Hoffmann et al., Phys. Rev. B 67, 220406(R) (2003).

[178] J. Camarero et al., Phys. Rev. Lett. 95, 057204 (2005).

[179] B. Beckmann et al., Phys. Rev. Lett. 91, 187201 (2003).

[180] A. Hoffmann, Phys. Rev. Lett. 93, 097203 (2004).

[181] M. Grimsditch et al., Phys. Rev. Lett. 90, 257201 (2003).

[182] J. McCord et al., Phys. Rev. B 78, 094419 (2008).

[183] C. H. Lai et al., Phys. Rev. B 64, 094420 (2001).

[184] J. Akerman et al., Phys. Rev. B 76, 144416 (2007).

[185] I. N. Krivorotov et al., Phys. Rev. B 65, 100402(R) (2002).

[186] Szunyogh et al, Phys. Rev B 79, 020403R (2009).

[187] R. Bali et al., J. Appl. Phys. 103, 053911 (2008).

[188] T. Mewes et al., Phys. Rev. B 68, 184418 (2003).

[189] J. Li et al., Phys. Rev. B 84, 094447 (2011).

[190] W. Kuch et al., Phys. Rev. B 75, 224406 (2007).

[191] R. P. Cowburn et al., Phys. Rev. Lett. 79, 4018 (1997).

[192] Q. Zhan et al, Phys. Rev. B 80, 094416 (2009).

[193] Q. Zhan et al, Appl. Phys. Lett. 91, 122510 (2007).

[194] Q. Zhan et al, New Journal of Physics 11, 063003 (2009).

[195] D. Ecija et al., Phys. Rev. B 77, 024426 (2008).

[196] P. Luches et al., Phys. Rev. B 81, 054431 (2010).

[197] H. H. Huang et al., Appl. Phys. Lett. 98, 072501 (2011).

[198] E. Arenholz et al., Appl. Phys. Lett. 87, 132501 (2005).

[199] S. Dubourg et al., Euro. Phys. J. B 45, 175 (2005).

[200] J. Demeter et al., Solid. State. Commun. 152, 292 (2012).

[201] Z. Wang et al., Phys. Rev. B 72, 054407 (2005).

[202] Review. J. I. Martin et al., J. Magn. Magn. Mater. 256, 449 (2003).

[203] Review. A. O. Adeyeye et al., J. Phys. D: Appl. Phys. 41, 153001 (2008).

[204] W. Zhang et al., J. Micromech. Microeng. 24, 093001 (2014).

[205] N. Singh et al., Nanotechnology 15, 1539 (2004).

[206] W. Zhang et al., J. Appl. Phys. 107, 09D724 (2010).

[207] W. Zhang et al., J. Appl. Phys. 113, 17B502 (2013).

[208] D. Tripathy et al., J. Appl. Phys. 107, 09D705 (2010).

[209] S. Goolaup et al., Eur. Phys. J. B 44, 259 (2005).

[210] S. H. Chung et al., Phys. Rev. B 71, 214430 (2005).

[211] T. Mewes et al., Phys. Rev. B 67, 104422 (2003).

[212] T. Gredig et al., Phys. Rev. B 74, 094431 (2006).

[213] Z. Ding et al., J. Appl. Phys. 113, 17B103 (2013).

[214] W. Zhang et al., J. Magn. Magn. Mater. 324, 3129 (2012).

[215] W. Zhang et al., J. Appl. Phys. 111, 07D712 (2012).

[216] P. Wadley et al, Science DOI: 10.1126/science.aab1031 (2016).

[217] P. Wadley et al, Nature Commun. 4, 2322 (2013).

[218] T. Jungwirth et al, Nature Nanotech. 11, 231 (2016).

[219] Review. A. H. MacDonald et al., Phil. Trans. R. Soc. A 369, 3098 (2011).

[220] A. S. Nunez et al., Phys. Rev. B 73, 214426 (2006).

[221] A. B. Shick et al., Phys. Rev. B 81, 212409 (2010).

[222] V. M. T. S. Barthem et al., Nat. Commun. 4, 2892 (2013).

[223] B. G. Park et al., Nat. Mater. 10, 347 (2011).

[224] J. B. Mendes et al., Phys. Rev. B 89, 140406(R) (2014).

[225] P. Merodio et al., Appl. Phys. Lett. 104, 032406 (2014).

[226] Y. Y. Wang et al, Phys. Rev. Lett. 109, 137201 (2012).

[227] C. Wang et al, Phys. Rev. X 4, 041034 (2014).

[228] I. Final et al, Nature Commun. 5, 4671 (2014). 
[229] X. Marti et al, Nature Mater. 13, 367 (2014).

[230] T. Moriyama et al, Appl. Phys. Lett. 107, 122403 (2015).

[231] M. I. D'yakonov and V. I. Perel, Sov. Phys. JETP Lett. 13, 467 (1971).

[232] Review. W. Zhang et al, J. App. Phys. 117, 172610 (2015).

[233] Review. A. Hoffmann, IEEE Trans. Magn. 49, 5172 (2013).

[234] L. Liu et al, Science 336, 555 (2012).

[235] R. Shindou et al., Phys. Rev. Lett. 87, 116801 (2001).

[236] H. Chen et al., Phys. Rev. Lett. 112, 017205 (2014).

[237] F. Freimuth et al., Phys. Rev. Lett. 105, 246602 (2010).

[238] D. Qu et al, Phys. Rev. B 92, 020418(R) (2015).

[239] J. Zelezny et al, Phys. Rev. Lett. 113, 157201 (2014).

[240] W. Zhang et al, Phys. Rev. Lett. 113, 196602 (2014).

[241] H. L. Wang et al., Phys. Rev. Lett. 112, 197201 (2014).

[242] W. Zhang et al, Phys. Rev. B 92, 144405 (2015).

[243] H. Nakayama et al, Phys. Rev. Lett. 110, 206601 (2013).

[244] C. Avci et al, Nature Phys. 11, 570 (2015).

[245] J. Kim et al, Phys. Rev. Lett. 116, 097201 (2016).

[246] J. H. Han et al, Phys. Rev. B 90, 144431 (2014).

[247] T. Lin et al, Phys. Rev. Lett. 113, 037203 (2014).

[248] Y. Wang et al, Adv. Mater. 27, 3196 (2015).

[249] S. Takei et al, Phys. Rev. B 90, 094408 (2014).

[250] H. L. Wang et al., Phys. Rev. Lett. 113, 097202 (2014).

[251] C. Hahn et al, EPL 108, 57005 (2014).

[252] H. L. Wang et al, Phys. Rev. B 91, 220410(R) (2015).

[253] T. Moriyama et al, Appl. Phys. Lett. 106, 162406 (2015).

[254] W. Lin et al, arXiv:1603.00931.

[255] L. Frangou et al, Phys. Rev. Lett. 116, 077203 (2016).

[256] R. Khymyn et al, arXiv:1511.05785.

[257] K. Chen et al, arXiv:1604.01714.

[258] Y. Ohnuma et al, Phys. Rev. B 87, 014423 (2013).

[259] S. M. Wu et al, Phys. Rev. Lett. 116, 097204 (2016).

[260] R. Cheng et al, Phys. Rev. Lett. 113, 057601 (2014).

[261] G. E. W. Bauer et al, Nature Mater. 11, 391 (2012).

[262] S. Seki et al, Phys. Rev. Lett. 115, 266601 (2015).

[263] S. M. Rezende et al, Phys. Rev. B 93, 014425 (2016).

[264] P. Ross et al, J. Appl. Phys. 118, 233907 (2015). 